\title{
Development
}

\section{Grp94 Regulates the Recruitment of Aneural AChR Clusters for the Assembly of Postsynaptic Specializations by Modulating ADF/Cofilin Activity and Turnover}

\author{
Zora Chui-Kuen Chan, ${ }^{*}$ Linyan Deng, ${ }^{*}$ and ${ }^{\circledR C h i}$ Wai Lee
}

https://doi.org/10.1523/ENEURO.0025-20.2020

School of Biomedical Sciences, Li Ka Shing Faculty of Medicine, The University of Hong Kong, Hong Kong

\begin{abstract}
Temperature is a physiological factor that affects neuronal growth and synaptic homeostasis at the invertebrate neuromuscular junctions (NMJs); however, whether temperature stress could also regulate the structure and function of the vertebrate NMJs remains unclear. In this study, we use Xenopus laevis primary cultures as a vertebrate model system for investigating the involvement of heat shock protein 90 (HSP90) family of stress proteins in NMJ development. First, cold temperature treatment or HSP90 inhibition attenuates the formation of aneural acetylcholine receptor (AChR) clusters, but increases their stability after they are formed, in cultured muscles. HSP90 inhibition specifically affects the stability of aneural AChR clusters and their associated intracellular scaffolding protein rapsyn, instead of causing a global change in cell metabolism and protein expression in Xenopus muscle cultures. Upon synaptogenic stimulation, a specific HSP90 family member, glucose-regulated protein 94 (Grp94), modulates the phosphorylation and dynamic turnover of actin depolymerizing factor (ADF)/cofilin at aneural AChR clusters, leading to the recruitment of AChR molecules from aneural clusters to the assembly of agrin-induced postsynaptic specializations. Finally, postsynaptic Grp94 knock-down significantly inhibits nerve-induced AChR clustering and postsynaptic activity in nervemuscle co-cultures as demonstrated by live-cell imaging and electrophysiological recording, respectively. Collectively, this study suggests that temperature-dependent alteration in Grp94 expression and activity inhibits the assembly of postsynaptic specializations through modulating ADF/cofilin phosphorylation and activity at aneural AChR clusters, which prevents AChR molecules from being recruited to the postsynaptic sites via actin-dependent vesicular trafficking, at developing vertebrate NMJs.
\end{abstract}

Key words: acetylcholine receptor; ADF/cofilin; Grp94; heat shock protein; neuromuscular junction; temperature stress

\section{Significance Statement}

Heat shock protein 90 (HSP90) is one of the most studied and abundant molecular chaperones of eukaryotic cells that protect proteins from cellular stress. Our study provides the first evidence showing that temperature-dependent alteration in the expression and activity of a specific HSP90 family member glucose-regulated protein 94 (Grp94) regulates the recruitment of aneural acetylcholine receptor (AChR) clusters for the assembly of postsynaptic specializations through actin depolymerizing factor (ADF)/cofilin-mediated vesicular trafficking at developing vertebrate neuromuscular junctions (NMJs). Given the recent identification of Grp94 and other endoplasmic reticulum (ER) chaperones as potential biomarkers for diagnosis of myasthenia gravis, an autoimmune NMJ disease, results of this study not only enhance our understanding on the fundamental mechanisms underlying NMJ development but also provide insights into the pathogenic mechanisms underlying ER stress response and NMJ disruption in neuromuscular diseases. 


\section{Introduction}

Synapses are the fundamental structures in the nervous system that enable efficient communication between neurons and their target cells. Neuromuscular junction (NMJ), a peripheral synapse, is formed between a motor neuron and a skeletal muscle fiber. Because of its accessibility and simplicity in structure, NMJ has served as a model synapse for elucidating the molecular mechanisms underlying synapse formation and maintenance in health, disease, and aging (Sanes and Lichtman, 2001; Li et al., 2018; Chan et al., 2020a). At developing NMJs, aggregation of acetylcholine receptors (AChRs) at the postsynaptic membranes represents an important step in neuromuscular synaptogenesis. Before nerve innervation, AChR molecules are both diffusely distributed throughout the muscle surface and spontaneously clustered in the form of AChR prepatterns (Yang et al., 2000, 2001; Lin et al., 2001). Upon synaptogenic induction, nerve-induced AChR clustering at the postsynaptic sites is believed to be contributed by the recruitment of both diffuse and prepatterned AChRs, as well as the local synthesis of AChR proteins at the sub-synaptic nuclei (Sanes and Lichtman, 2001). The postsynaptic specializations at NMJs are associated with dense networks of stable filamentous actin (F-actin) structures at the cell cortex, which mediate AChR cluster formation and redistribution through rapsyn (Dai et al., 2000; Borges and Ferns, 2001; Dobbins et al., 2008). A previous study has demonstrated that actin depolymerizing factor (ADF)/cofilin-mediated actin dynamics regulate the vesicular trafficking of AChRs at developing NMJs (Lee et al., 2009). These findings suggest a novel ADF/cofilin-dependent transcytosis mechanism underlying the redistribution of aneural AChR clusters for the assembly of synaptic AChR clusters at NMJs. However, the mechanistic regulation of $A C h R$ redistribution from aneural to synaptic clusters at developing NMJs remains unclear.

Heat shock proteins (HSPs) are molecular chaperones that show remarkable sequence homology across the phylogenetic spectrum from a unicellular organism, Saccharomyces cerevisiae, to a multicellular organism, mammal. These stress proteins are grouped into major families according to their

Received January 26, 2020; accepted July 27, 2020; First published August 3, 2020.

The authors declare no competing financial interests.

Author contributions: C.W.L. designed research; Z.C.-K.C., L.D., and C.W.L. performed research; C.W.L. contributed unpublished reagents/analytic tools; Z.C.-K.C., L.D., and C.W.L. analyzed data; C.W.L. wrote the paper.

This work was partly supported by the Early Career Grant 27102316 and General Research Fund Grants 17100718 and 17100219 from the Research Grants Council of Hong Kong, the Health and Medical Research Fund Grant 04151086 from the Food and Health Bureau of Hong Kong, and Seed Fund Programme for Basic Research from The University of Hong Kong Grants 201601159003, 201711159098, and 201811159078 (to C.W.L.).

*Z.C.-K.C. and L.D. contributed equally to this work.

Acknowledgements: We thank the assistance of Proteomics and Metabolomics Core and Imaging Core, Centre for PanorOmic Sciences at HKU Medicine.

Correspondence should be addressed to Chi Wai Lee at chiwai.lee@hku.hk. https://doi.org/10.1523/ENEURO.0025-20.2020 Copyright @ 2020 Chan et al.

This is an open-access article distributed under the terms of the Creative Commons Attribution 4.0 International license, which permits unrestricted use, distribution and reproduction in any medium provided that the original work is properly attributed. approximate molecular weight in $\mathrm{kDa}$. Among them, HSP90 is a highly abundant and ubiquitous molecular chaperone, which plays an essential role in many different processes to maintain cellular homeostasis under stressful conditions (Schopf et al., 2017). The HSP90 family includes cytosolic HSPs (HSP9O $\alpha$ and HSP90 $\beta$ ), endoplasmic reticulum (ER)resident glucose-regulated protein 94 (Grp94), and mitochondrial-specific tumor necrosis factor receptor-associated protein-1 (TRAP-1). Grp94, encoded by the HSP90B1 gene, shares many biochemical features with other HSP90 proteins (Csermely et al., 1998; Marzec et al., 2012). It is believed that Grp94 can escape ER retention and retrieval in cells under ER stress (Gutiérrez and Simmen, 2014). Intriguingly, a previous study suggested that Grp94 can also be found in the cell surface of $\mathrm{C} 2 \mathrm{C} 12$ myotubes, in which Grp94 phosphorylation mediated by the Src kinase Fyn promotes the chaperone export from the ER during the early phase of myoblast differentiation (Frasson et al., 2009). Given that the cytosolic $\operatorname{HSP} 90 \beta$ is known to regulate AChR cluster formation and maintenance through modulating rapsyn turnover (Luo et al., 2008), whether the ER-resident HSP90 family member, Grp94, is involved in NMJ development remains unknown.

In this study, we first show that temperature stress upregulates the mRNA transcript levels of both $\operatorname{HSP} 90 \beta$ and Grp94, but down-regulates the protein level of only Grp94, in cultured Xenopus muscle cells. Interestingly, pharmacological inhibition of HSP90 activity by 17-(allylamino)-17demethoxygeldanamycin (17-AAG) or molecular manipulation of endogenous Grp94 expression suppresses the formation of aneural AChR clusters and increases the stability of aneural AChR clusters after they are formed. Upon synaptogenic stimulation, the recruitment of preexisting AChRs to agrin-induced AChR clusters is significantly reduced in wild-type (WT) muscle cells treated with 17-AAG and in Grp94 knock-down muscle cells. Interestingly, 17AAG treatment accelerates the turnover of green fluorescent protein-tagged Xenopus ADF/cofilin (GFP-XAC) at both perforated and AChR-rich regions of aneural clusters resembling the dynamic turnover of GFP-XAC inactive (S3E) mutant, suggesting that HSP90 regulates dephosphorylation and activation of ADF/cofilin. Finally, nerve-induced AChR clustering and synaptic functions are impaired in chimeric nerve-muscle co-cultures containing Grp94 knock-down muscles and WT neurons, indicating the essential roles of postsynaptic Grp94 in regulating synaptic structure and function of developing NMJs. Together, our study suggests that temperature-dependent alteration in Grp94 expression and activity regulates the recruitment of AChR molecules from aneural to agrin-induced synaptic clusters through modulating ADF/cofilin phosphoregulation to mediate actin-dependent vesicular trafficking at developing NMJs.

\section{Materials and Methods}

\section{Embryo microinjection and primary culture from Xenopus embryos}

Adult female frogs (Xenopus 1, RRID: XEP_Xla100) were injected with 1000 I.U. human chorionic gonadotropin (hCG; 
Sigma, catalog \#CG10) with $0.1 \%$ BSA to induce ovulation. After fertilization in vitro, embryos were maintained in Holtfreter's solution (v/v; $60 \mathrm{~mm} \mathrm{NaCl}, 0.6 \mathrm{~mm} \mathrm{KCl,} 0.9 \mathrm{~mm}$ $\mathrm{CaCl}_{2}$, and $0.2 \mathrm{~mm} \mathrm{NaHCO}$; $\mathrm{pH}$ 7.4). A total of $20-100 \mathrm{pg}$ of DNA constructs encoding either WT or phosphorylation mutant forms (S3A and S3E) of GFP-XAC (gifts from Dr. James Bamburg, Colorado State University) were microinjected into one blastomere of one- or two-cell stage Xenopus embryos with an oocyte injector. GFP-expressing embryos were screened for primary culture preparation. Myotomal muscle tissues and neural tubes were isolated from stage 19-22 Xenopus embryos after enzymatic digestion with $1 \mathrm{mg} / \mathrm{ml}$ collagenase (Sigma-Aldrich, catalog \#C98191G), followed by dissociation with calcium-magnesium-free solution. Dissociated muscle cells were then plated on glass bottom dishes or glass coverslips coated with entactin-collagen IV-laminin (ECL) cell attachment matrix (Merck Millipore, catalog \#08-100). The ECL coating was performed by incubating the coverslips or dishes with $10 \mu \mathrm{g} / \mathrm{ml} \mathrm{ECL} \mathrm{in}$ 10\% Leibovitz's L-15 medium (Sigma-Aldrich, catalog \#L4386) at $37^{\circ} \mathrm{C}$ for $3 \mathrm{~h}$. The coating was later washed with PBS solution (137 mM NaCl, $2.7 \mathrm{~mm} \mathrm{KCl,} 10 \mathrm{~mm} \mathrm{Na}_{2} \mathrm{HPO}_{4}$, $1.8 \mathrm{mM} \mathrm{KH}_{2} \mathrm{PO}_{4}$ ), followed by Steinberg's solution [60 mM $\mathrm{NaCl}, 0.67 \mathrm{~mm} \mathrm{KCl}, 0.35 \mathrm{~mm} \mathrm{Ca}\left(\mathrm{NO}_{3}\right)_{2}, 0.83 \mathrm{~mm} \mathrm{MgSO}_{4}$, and 10 mм HEPES; pH 7.4]. Cells were cultured in medium containing $87 \% \quad(\mathrm{v} / \mathrm{v})$ Steinberg's solution, $10 \% \quad(\mathrm{v} / \mathrm{v})$ Leibovitz's L-15 medium, 1\% fetal bovine serum (Invitrogen, catalog \#10270), 1\% penicillin/streptomycin (Thermo Fisher Scientific, catalog \#15140122), and $1 \%$ gentamicin sulfate (Thermo Fisher Scientific, catalog \#15750060). Muscle cells were kept at $22^{\circ} \mathrm{C}$ for at least $24 \mathrm{~h}$ to allow cell attachment and aneural AChR cluster formation before treatments, if any. To make nerve-muscle or bead-muscle coculture, dissociated spinal neurons or polystyrene latex beads coated with agrin (R\&D Systems, catalog \#550-AG-100/CF) were added in 2-d-old muscle cultures and maintained for $1 \mathrm{~d}$ before imaging. All the experiments involving Xenopus frogs and embryos were performed in accordance with The University of Hong Kong animal care committee's regulations.

\section{Morpholino-mediated knock-down of endogenous proteins}

Knock-down of endogenous proteins in Xenopus was achieved by embryonic injection of antisense morpholino oligonucleotides (MO; Gene Tools), which bind to the target mRNA sequence that block its protein translation. The following $M O$ sequences were used in this study: Xenopus Grp94 MO: 5'-GACCGATTGCCCAAAACTTCCT CAT-3', Xenopus HSP90 $\beta$ MO: 5'-CATTGTGGGCAA CTTCTGGCATC-3', and Control MO: 5'-CCTCT TACCT CAGTT ACAAT TTATA-3'. To visualize the presence of $\mathrm{MO}$ in the microinjected embryos, MOs were co-injected with Alexa Fluor 488-conjugated dextran (Thermo Fisher Scientific, catalog \#D22910) as a cell lineage tracer. The effectiveness of MO-mediated knock-down of endogenous proteins was validated by Western blot analyses.

\section{Quantitative real-time RT-PCR analysis of HSP90 expression}

Total RNA was extracted using the TRIzol reagent from 2-d-old Xenopus muscle cultures incubated at $22^{\circ} \mathrm{C}, 15^{\circ}$
$\mathrm{C}$, or $10^{\circ} \mathrm{C}$, respectively. Isolated RNA samples were treated with DNase (Thermo Fisher Scientific, catalog \#EN0521) to remove genomic DNA. An equal amount of RNA from samples was reverse transcribed into cDNA with High-Capacity cDNA Reverse Transcription kit (Thermo Fisher Scientific, catalog \#4368814) and qPCR was performed using CXF96 Touch together with SYBR Premix Ex Taq (Takara Bio, catalog \#RR420A). Data were acquired and analyzed with CFX Manager (Bio-Rad, RRID: SCR_017251). Primers are listed below:

HSP90 $\alpha$ forward: 5' -TCTGACTGACCCAAGCAAAC-3'; HSP90 $\alpha$ reverse: $5^{\prime}$-GCCTGCAAAGCCTCCATAAA-3'; HSP90 $\beta$ forward: 5'-CTATGATTGATACCGGAATT-3'; HSP90 $\beta$ reverse: 5'-CATATTGCTCATCATCATTG-3'; Grp94 forward: 5'-CACTGATGACCCTCGTGGTG-3'; Grp94 reverse: 5'-AGGGGCTCCTCTACTGTCTC-3'; TRAP-1 forward: 5'-CCCAGGGACAAAGGTTGTGA-3'; TRAP-1 reverse: 5'-TCATGCTGCCATTCCCCAAT-3'; GAPDH forward: 5'-GTGTATGTGGTGGAATCT-3';

GAPDH reverse: 5'-AAGTTGTCGTTGATGACCTTT GC-3'.

\section{Pharmacological treatment}

For experiments studying the effect of HSP90 on aneural AChR cluster formation, muscle cultures were pretreated with different concentrations $(0.1 \mathrm{~nm}, 0.25 \mathrm{nM}$, or $1 \mathrm{~nm}$ ) of 17-AAG (ApexBio, catalog \#A4054-10). To investigate the effect of HSP90 in the remodeling of AChR clusters, 2-d-old muscle cultures were treated with 17-AAG (1 nM) or PU-WS13 (15 $\mu \mathrm{m}$; ApexBio, catalog \#B5885). For experiments investigating the contribution of aneural AChR clusters to agrin-induced synaptic AChR clusters, 2-d-old muscle cells were treated with 17-AAG (1 nM) from $1 \mathrm{~h}$ before photobleaching experiments. For the experiments investigating the nerve-induced AChR clusters, PU-WS13 (15 $\mu \mathrm{M})$ was applied to 2-d-old muscle cultures from $1 \mathrm{~h}$ before spinal neurons plating. For experiment investigating inhibition of HSP90 activity on rapsyn localization in aneural AChR clusters, 2-d-old muscle cultures were treated with $1 \mathrm{~nm} 17-\mathrm{AAG}$ from $1 \mathrm{~h}$ before agrin bead addition. For experiments investigating the HSP9O and Grp94 activity in rapsyn localization and AChR internalization, 2-d-old muscle cultures were treated with $1 \mathrm{~nm} 17$ AAG or $15 \mu \mathrm{M}$ PU-WS13 from $1 \mathrm{~h}$ before plating spinal neurons or adding agrin beads.

\section{Labeling of different AChR pools in cultured muscle cells}

To differentially identify the preexisting and newly synthesized AChRs, surface AChRs in 2-d-old muscle cultures were first labeled with $0.1 \mu \mathrm{m}$ tetramethylrhodamine (Rh)-conjugated (Thermo Fisher Scientific, catalog \#T1175), Alexa Fluor 488-conjugated (488; Thermo Fisher Scientific, catalog \#B13422), or Alexa Fluor 647-conjugated (647; Thermo Fisher Scientific, catalog \#B35450) $\alpha$-bungarotoxin (BTX) for $45 \mathrm{~min}$, followed by extensive washing with culture medium. Cells were then saturated with a high dose of unconjugated BTX $(6 \mu \mathrm{M}$, Thermo Fisher Scientific, catalog \#B1601) for 30 min, followed by 
extensive washing with culture medium. Newly synthesized and inserted AChRs were then labeled with $1 \mu \mathrm{M}$ 488-BTX or 647-BTX at different timepoints, as specified. For live imaging, glass coverslips with culture cells were mounted on sealed live chamber containing culture medium. To determine the density of surface AChRs, cultured muscle cells were first labeled with $0.2 \mu \mathrm{m}$ biotin-XX-conjugated BTX (biotin-BTX; Thermo Fisher Scientific, catalog \#B1196) for $45 \mathrm{~min}$, then fixed with $4 \%$ paraformaldehyde in PBS for 15 min and permeabilized with $0.5 \%$ Triton X-100 in PBS for $10 \mathrm{~min}$. The samples were then incubated with 0.16 $\mu \mathrm{M}$ Qdot 655 (QD)-conjugated streptavidin (Thermo Fisher Scientific, catalog \#Q10143MP) for $10 \mathrm{~min}$. For experiments testing the possibility of AChR photo-dissipation, surface AChRs were labeled with $1 \mu \mathrm{m}$ Alexa Fluor 594-BTX (594BTX; Thermo Fisher Scientific, catalog \#B13423) or rhodamine-BTX (Rh-BTX) for $45 \mathrm{~min}$, followed by extensive washing with culture medium. After 6 or $24 \mathrm{~h}$, newly inserted surface AChRs were labeled with $1 \mu \mathrm{m}$ 488-BTX for $45 \mathrm{~min}$. To differentially identify surface and internal AChRs, cells were first fixed with $4 \%$ paraformaldehyde in PBS for $15 \mathrm{~min}$. Surface AChRs were labeled with $0.1 \mu \mathrm{m}$ Rh-BTX for $45 \mathrm{~min}$, followed by saturation with $6 \mu \mathrm{m}$ unconjugated BTX for 30 min. After cell permeabilization with $0.5 \%$ Triton X100, internal AChRs were labeled with $0.1 \mu \mathrm{M}$ 488-BTX for $45 \mathrm{~min}$. Coverslips were then mounted on glass slides with the anti-bleaching reagent fluoromount-G (Thermo Fisher Scientific, catalog \#00-4958-02) for later observation.

\section{Fixation and immunostaining of cells}

Xenopus muscle cultures were fixed with $4 \%$ paraformaldehyde in PBS for $15 \mathrm{~min}$. After extensive washing with PBS, fixed cells were permeabilized with $0.5 \%$ Triton X-100 in PBS, followed by blocking with 2\% BSA at room temperature for $2 \mathrm{~h}$ or at $4^{\circ} \mathrm{C}$ overnight. Cells were incubated with primary antibodies, including XAC (1:500, a gift from Dr. James Bamburg, Colorado State University), p34Arc/ARPC2 (1:100; EMD Millipore, catalog \#07-227, RRID: AB_310447), rapsyn (1:100; Affinity Bioreagent, catalog \#MA1-746, RRID: AB 2177611), or vinculin (1:250; SigmaAldrich, catalog \#V4505, RRID: AB_477617) for $2 \mathrm{~h}$, followed by fluorophore-conjugated secondary antibodies (Thermo Fisher Scientific, catalog \#A-11029, RRID: AB_2534088; catalog \#A-21206, RRID: AB_2535792) for $45 \mathrm{~min}$. Coverslips were then mounted on glass slides with the antibleaching reagent fluoromount-G for later observation.

\section{Quantitative metabolomics}

\section{Extraction and derivatization of polar metabolites}

A total of $1000 \mu \mathrm{l}$ methanol/water (80\%, v/v) with $200 \mathrm{ng}$ norvaline internal standard was added to the samples. The samples were homogenized after two cycles of sonication at $10 \mu \mathrm{m}$ for $20 \mathrm{~s}$ on ice and $10-\mathrm{s}$ pause time. The samples were centrifuged for $5 \mathrm{~min}$ at $16,000 \times g$ at $4^{\circ} \mathrm{C}$. A total of $500 \mu$ l supernatant was dried under a gentle stream of nitrogen at room temperature for derivatization. The dried residue was redissolved and derivatized for $2 \mathrm{~h}$ at $37^{\circ} \mathrm{C}$ in $40 \mu \mathrm{l}$ methoxylamine hydrochloride $(30 \mathrm{mg} / \mathrm{ml}$ in pyridine), followed by trimethylsilylation for $1 \mathrm{~h}$ at $37^{\circ} \mathrm{C}$ in $70 \mu \mathrm{l}$ MSTFA with $1 \%$ TMCS. Up to $1 \mu$ l sample was injected for gas chromatography-mass spectrometry (GCMS)/MS analysis.

\section{Extraction and transesterification of fatty acid metabolites}

A total of $100 \mu \mathrm{l}$ chloroform with $20 \mu \mathrm{g} \mathrm{C19:0} \mathrm{fatty} \mathrm{acid}$ internal standard was spiked to the samples. The samples were homogenized after two cycles of sonication at 10 $\mu \mathrm{m}$ for $20 \mathrm{~s}$ on ice and 10-s pause time. The sample was centrifuged for $5 \mathrm{~min}$ at $16,000 \times g$ at $4^{\circ} \mathrm{C}$. The pellet was separated and stored at $-80^{\circ} \mathrm{C} ; 1 \mathrm{ml}$ methanol and $50 \mu \mathrm{l}$ concentrated hydrochloric acid $(35 \%, \mathrm{w} / \mathrm{w})$ were added to the sample. The solution was overlaid with nitrogen and the tube was tightly closed. After vortexing, the tube was heated at $100^{\circ} \mathrm{C}$ for $1 \mathrm{~h}$. Once cooled to room temperature, $1 \mathrm{ml}$ hexane and $1 \mathrm{ml}$ water were added for FAMEs extraction. The tube was vortexed and after phase separation, up to $1 \mu$ l the hexane phase was injected for GC-MS analysis.

\section{Data acquisition}

GC-MS chromatogram was acquired in SCAN and MRM mode in an Agilent 7890B GC-Agilent 7010 Triple Quadrapole Mass Spectrometer system. For polar metabolites, the samples were separated through an Agilent DB-5MS capillary column $(30 \times 0.25 \mathrm{~mm}$ ID, $0.25-\mu \mathrm{m}$ film thickness) under constant flow at $1 \mathrm{ml} \mathrm{min}{ }^{-1}$. Characteristic quantifier and qualifier transitions were monitored in MRM mode during the run. For fatty acid metabolites, the samples were separated through an Aligent DB-23 capillary column $(60 \times 0.25 \mathrm{~mm} \mathrm{ID}, 0.15-\mu \mathrm{m}$ film thickness) under constant pressure at 33.4 psi. Characteristic fragment ions $(\mathrm{m} / \mathrm{z} 55$, $67,69,74,79,81,83,87,91,93,95,96,97,115,127,143$ ) were monitored in SIM mode throughout the run. Mass spectra from $\mathrm{m} / \mathrm{z}$ 50-350 were acquired in SCAN mode. Principal component analyses (PCAs) of polar metabolites and fatty acids was conducted with the determined data peaks using MetaboAnalyst 4.0 (Chong et al., 2019).

\section{Protein synthesis assay}

To determine the effects of HSP90 inhibition on protein synthesis in cultured Xenopus muscle cells, 1 nм 17-AAG was added to culture medium before cell plating. For the positive control, 4-d-old muscle cells were treated with 25 $\mu \mathrm{M}$ cycloheximide (CHX; ApexBio, catalog \#A8244-1000) for $2 \mathrm{~h}$ before adding O-propargyl-puromycin (OPP) reagent (Thermo Fisher Scientific, catalog \#C10456). After $30 \mathrm{~min}$, cells were fixed with $4 \%$ paraformaldehyde in PBS for $15 \mathrm{~min}$ and permeabilized with $0.5 \%$ Triton X-100 in PBS, followed by OPP detection according to manufacturer's instructions.

\section{Immunoblotting}

To validate the effectiveness of MO-mediated knockdown of endogenous proteins, the dorsal parts from Xenopus embryos at Nieuwkoop and Faber stage 19-22 were homogenized in RIPA buffer (Thermo Fisher Scientific, catalog \#89900) containing 1\% protease inhibitor cocktail and 1\% EDTA. For experiments investigating the effects of temperature on HSP90 $\beta$ and Grp94 
expression, 2-d-old cultured muscle cells incubated at $22^{\circ} \mathrm{C}$ or $10^{\circ} \mathrm{C}$ were scraped and homogenized in RIPA buffer containing 1\% protease inhibitor cocktail and 1\% EDTA. Protein lysates were obtained from the supernatant after high-speed centrifugation $(15,000 \times g)$. The concentration of protein lysates was determined by BCA protein assay kit (Thermo Fisher Scientific, catalog \#23227); 10$30 \mu \mathrm{g}$ protein lysates, Pierce lane marker non-reducing sample buffer $(25 \% \mathrm{v} / \mathrm{v}$ to sample buffer, Thermo Fisher Scientific, catalog \#39001), and $\beta$-mercaptoethanol (5\%; $\mathrm{v} / \mathrm{v}$ to sample buffer, Bio-Rad, catalog \#1610710) were loaded and separated on a 10\% TGX Stain-Free polyacrylamide gel, followed by transferring onto polyvinylidene difluoride (PVDF) membranes. The blot was blocked by immersing in 5\% non-fat milk containing TBST for $1 \mathrm{~h}$ at room temperature. The blots were probed for the following primary antibodies: Grp94 (1:1000; Thermo Fisher Scientific, catalog \#36-2600, RRID: AB_2533246), HSP90 $\beta$ (1:1000; Thermo Fisher Scientific, catalog \#37-9400, RRID: AB_ 2533349), or $\beta$-tubulin (1:1000; DSHB, catalog \#EVII-s, RRID: AB_528499) at $4^{\circ} \mathrm{C}$ overnight, followed by HRP-conjugated secondary antibodies (1:5000; Thermo Fisher Scientific, catalog \#G-21040, RRID: AB_2536527; catalog \#G-21234, RRID: AB_2536530) at room temperature for $1 \mathrm{~h}$. Signals were visualized using Pierce enhanced chemiluminescence substrate (Thermo Fisher Scientific, catalog \#32106), and image acquisition was performed with Image Lab 6.0.1 (Bio-Rad) by ChemiDoc XRS + System (Bio-Rad).

\section{Electrophysiological recordings}

For cultures used for electrophysiological recordings, myotubes and spinal neurons were plated together on plain glass coverslips. Spontaneous synaptic currents (SSCs) were recorded from myoballs in 1-d-old nervemuscle coculture by whole-cell patch clamp recording. The cultures were perfused with the recording solution, containing $140 \mathrm{~mm} \mathrm{NaCl}, 5 \mathrm{~mm} \mathrm{KCl}, 1 \mathrm{~mm} \mathrm{CaCl}_{2}, 1 \mathrm{~mm}$ $\mathrm{MgCl}_{2}$, and $10 \mathrm{~mm}$ HEPES; $\mathrm{pH}$ 7.4. Glass micropipettes (Sutter instrument) with 1-3 $\mathrm{M} \Omega$ was filled with intrapipette solution containing $145 \mathrm{~mm} \mathrm{KCl}, 1 \mathrm{~mm} \mathrm{NaCl}, 1 \mathrm{~mm}$ $\mathrm{MgCl}_{2}, 1 \mathrm{~mm} \mathrm{Mg}-\mathrm{ATP}$, and $10 \mathrm{~mm}$ HEPES; pH 7.2. Muscle cells were voltage clamped at $-70 \mathrm{mV}$. All data were obtained using the MultiClamp 700B amplifier (Molecular Devices). The signals were filtered at $2 \mathrm{kHz}$ and sampled at $20 \mathrm{kHz}$ using Digidata 1440A (Molecular Devices, RRID: SCR_018455). The frequency of SSCs was defined as the number of events per second. The frequency, amplitude, rise time, decay time, and inter-event intervals of SSCs were analyzed using MiniAnalysis Program v6.0.3 (Synaptosoft, RRID: SCR_002184).

\section{Fluorescence microscopy}

The fluorescent images were acquired on inverted epifluorescence microscopes (IX81 or IX83; Olympus) with an oil immersion $60 \times$ NA 1.42 objective lens or with a $20 \times$ NA 0.5 objective lens. Images were captured by iXon EMCCD camera (Andor) using the cell ${ }^{\mathrm{a}} \mathrm{R}$ software (Olympus) on IX81, or by ORCA-Flash4.0 LT+ digital CMOS camera (Hamamatsu) using MicroManager software v.1.4 (Open
Imaging, RRID: SCR_016865) on IX83. Quantitative measurements of fluorescence images were performed by ImageJ software (National Institute of Health, RRID: SCR_003070).

Fluorescence images of QD-labeled single AChR molecules were acquired on a super-resolution structured illumination microscopy (SR-SIM Elyra S1; Carl Zeiss) with an oil immersion $60 \times$ NA 1.4 objective lens. Images were obtained by a sCMOS camera (PCO Edge) with cooling system using ZEN 2.3 software (Carl Zeiss, RRID: SCR 018163).

Photobleaching of aneural AChR clusters was performed on a confocal microscope (LSM 800; Carl Zeiss) using $20 \times$ NA 0.8 objective lens and Diode laser line (488 or $561 \mathrm{~nm}$ ) with $100 \%$ laser intensity. Identical settings were applied in all photobleaching experiments. A fluorescence image was taken immediately after photobleaching to ensure the complete photobleaching of AChR signals.

Fluorescence recovery after photobleaching (FRAP) experiments were performed in total internal reflection fluorescence (TIRF) mode using Axio TIRF unit fitted on an inverted microscope equipped with oil immersion $100 \times$ NA 1.46 DIC objective lens. Photobleaching was performed by using Sapphire laser line $(488 \mathrm{~nm})$ with $50 \%$ laser intensity. Images were captured through MetaMorph (Molecular Devices, RRID: SCR_002368) by Evolve 512 EMCC camera (Photometrics). Identical settings were applied in all photobleaching experiments. To obtain the baseline of fluorescence intensity before photobleaching, 10 images at 1-s interval were taken in muscle cells expressing WT or mutant forms of GFPXAC before photobleaching. After photobleaching, time-lapse images were taken at 1-s interval until the fluorescence intensity has reached the plateau level.

\section{Statistical analysis}

All data from this study were collected from at least three replicates in independent experiments. Prism 7.0 (GraphPad) was used for statistical analyses. Detailed statistical results, including the exact $p$ values, are provided in Table 1.

\section{Results}

\section{HSP90 expression and activity regulate AChR cluster formation and stability}

As Xenopus primary cultures can be maintained in a range of different temperatures, this unique feature allows us to investigate whether temperature stress regulates NMJ development. Here, we incubated dissociated Xenopus myotomal tissues plated on coated substratum containing laminin under different culture temperatures (Fig. 1A). At the normal culture temperature at $22^{\circ} \mathrm{C}$ (Peng et al., 1991), we detected an increasing percentage of cultured muscle cells with spontaneously formed aneural AChR clusters over the first $3 d$ in culture (Fig. 1B). Interestingly, the formation of aneural AChR clusters was greatly reduced in muscle cultures incubated at lower temperatures $\left(15^{\circ} \mathrm{C}\right.$ or $\left.10^{\circ} \mathrm{C}\right)$, which showed a temperature-dependent response during the entire 4-d culture period (Fig. $1 A, B)$. Notably, cultured muscle cells at lower 


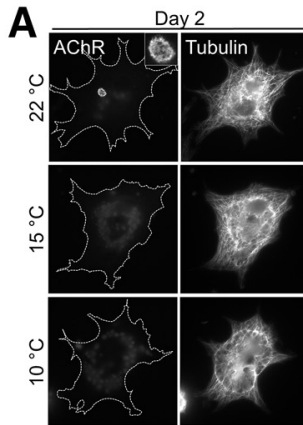

D

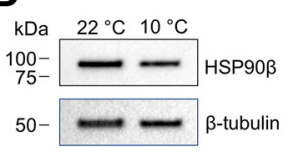

$\mathbf{F}$

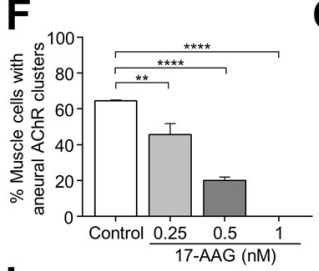

G

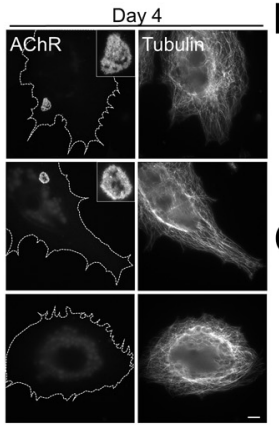

B

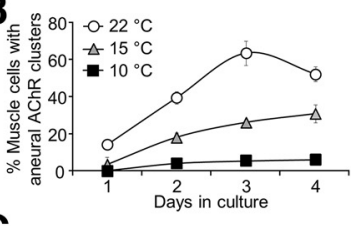

C

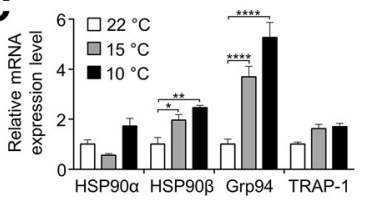

E
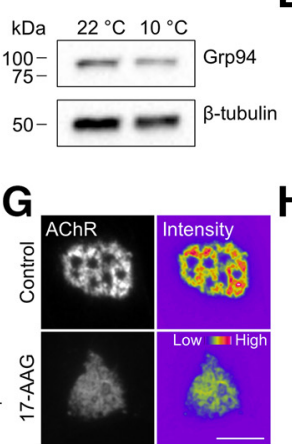

H
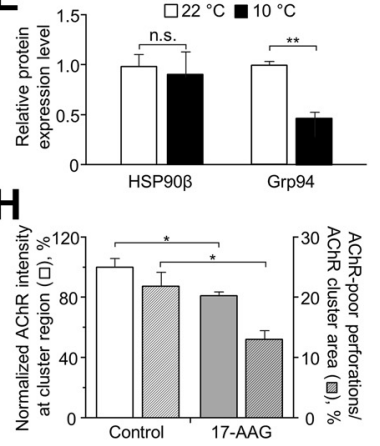

$\mathbf{K}$
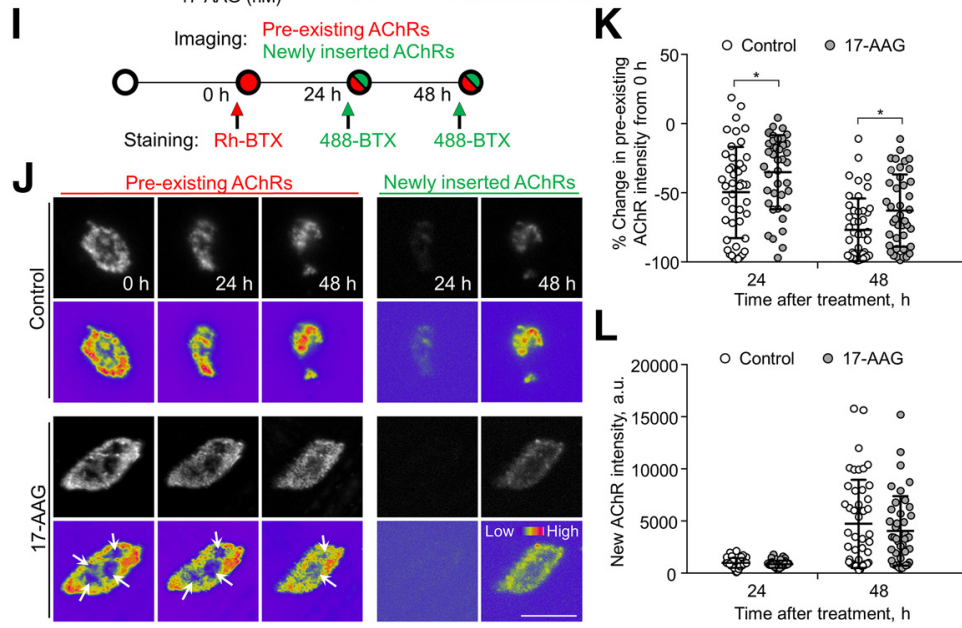

Figure 1. Temperature stress-modulated expression and pharmacological inhibition of HSP90 regulate the formation and stability of aneural AChR clusters. A, Representative images showing the inhibition of aneural AChR cluster formation in cultured Xenopus muscle cells treated with lower temperatures. Tubulin immunostaining indicated that cytoskeletal structures were largely unaffected in muscle cells cultured at different temperatures, ranging from $10-22^{\circ} \mathrm{C}$. B, Quantification showing the percentage of cultured muscle cells with bottom aneural AChR clusters at different culturing temperatures over $4 \mathrm{~d} ; n=150$ cells in each condition from three independent experiments. C, Quantification showing the relative mRNA levels of HSP90 $\alpha$, HSP90 $\beta$, Grp94, and TRAP-1 in 2-d-old Xenopus muscle cells cultured at different temperatures; $n=3$ independent experiments. $\boldsymbol{D}, \boldsymbol{E}$, Western blot analysis $(\boldsymbol{D})$ and quantification $(\boldsymbol{E})$ showing the protein expression level of $\mathrm{HSP} 90 \beta$ and Grp94 in Xenopus muscle cells cultured at $22^{\circ} \mathrm{C}$ or $10^{\circ} \mathrm{C}$ for $2 \mathrm{~d}$. $\beta$-Tubulin was used as the loading control for normalization. $\boldsymbol{F}$, Quantification showing the dose-dependent effects of 17-AAG on aneural AChR cluster formation in cultured Xenopus muscle cells; $n=191$ (Control), $n=198$ (0.25 nм 17-AAG), $n=199$ (0.5 nм 17AAG), and $n=200$ (1 nм 17-AAG) muscle cells from four independent experiments. G, Representative images showing the organization and intensity of aneural AChR clusters in response to 17-AAG treatment. 8-bit pseudo-color images highlight the relative fluorescence intensity of AChR clusters in different conditions. $\boldsymbol{H}$, Quantification showing the effects of 17-AAG on the intensity and complexity of aneural AChR clusters; $n=55$ (Control) and $n=44$ (17-AAG) muscle cells from three independent experiments for fluorescence intensity measurement (left $y$-axis); $n=76$ (Control) and $n=48$ (17-AAG) muscle cells from four independent experiments for cluster complexity measurement (right $y$-axis). I, Schematic diagram illustrating the differential labeling procedure to identify preexisting (red) and newly inserted (green) AChRs with BTX conjugated with different fluorophores. J, Representative sets of timelapse images showing the topological changes and fluorescence intensity of pre-existing (left panels) and newly inserted (right panels) AChRs at aneural clusters in control (top panels) or 17-AAG-treated (bottom panels) muscle cells. Arrows indicate the progressive reduction of perforated area in aneural AChR clusters. 8-bit pseudo-color images highlight the change in the fluorescence intensity of the same aneural AChR clusters over $48 \mathrm{~h}$ with or without $17-\mathrm{AAG}$ treatment. $\boldsymbol{K}, \boldsymbol{L}$, Individual value plots showing the 
continued

percentage change in the fluorescence intensity of pre-existing $(\boldsymbol{K})$ and newly inserted $(\boldsymbol{L}) \mathrm{AChRs}$ in the same aneural AChR clusters at different time-points between control and 17-AAG-treated cells; $n=46$ (Control) and $n=41$ (17-AAG) muscle cells from three independent experiments. Scale bars: $10 \mu \mathrm{m}$. Data are shown as mean \pm SEM $(\boldsymbol{B}, \boldsymbol{C}, \boldsymbol{E}, \boldsymbol{F}, \boldsymbol{H})$ or mean \pm SD $(\boldsymbol{K}, \boldsymbol{L})$. Two-way ANOVA with Tukey's multiple comparisons test $(\boldsymbol{B}, \boldsymbol{C})$, Student's $t$ test $(\boldsymbol{E}, \boldsymbol{H})$, one-way ANOVA with Tukey's multiple comparison test $(\boldsymbol{F})$, and two-way ANOVA with Sidak's multiple comparisons test $(\boldsymbol{K}, \boldsymbol{L})$. *, **, and $* * * *$ represent $p \leq 0.05,0.01$, and 0.0001 , respectively. n.s.: non-significant.

temperatures did not show any obvious changes in the cytoskeletal organization, as evidenced by the integrity of microtubule networks throughout the cells across different temperature groups (Fig. 1A). These data suggest that temperature stress causes a specific inhibition on aneural AChR cluster formation, rather than some non-specific cellular structure defects, in Xenopus muscle cultures.

The expression of HSP family of proteins could be induced in cells under stressful conditions, including temperature changes (Richter et al., 2010). A previous study showed that the molecular chaperone $\mathrm{HSP} 90 \beta$ plays a role in AChR cluster formation and maintenance by regulating rapsyn turnover (Luo et al., 2008), suggesting the involvement of stress proteins in regulating NMJ development. To examine if the expression of stress proteins in cultured Xenopus muscle cells is affected by low-temperature treatment, we first performed quantitative real-time PCR to examine mRNA levels of several HSP90 family members in muscle cells incubated at different temperatures (Fig. 1C). Surprisingly, the expression of $\mathrm{HSP} 90 \alpha$, the inducible isoform of HSP90, was not significantly changed in low-temperature treatments. Instead, we detected a temperature-dependent increase in the expression of another two HSP90 family members, HSP90 $\beta$ and Grp94, in $10^{\circ} \mathrm{C}$ muscle cultures, which exhibited 2.5-fold and 5.2-fold increase in HSP90 $\beta$ and Grp94 mRNA levels, respectively, compared with that in $22^{\circ} \mathrm{C}$ muscle cultures. On the other hand, the expression level of mitochondrial-specific HSP90 protein TRAP-1 remained unchanged in different temperature groups. We then performed Western blot analyses to further investigate whether the protein expressions of HSP90 $\beta$ and Grp94 are affected by low-temperature treatment (Fig. 1D,E). Surprisingly, in contrast to the increase in HSP90 $\beta$ and Grp94 mRNA levels by temperature stress, the protein level of Grp94, but not HSP90 $\beta$, was significantly reduced by $53.3 \pm 0.06 \%$ in cultured muscle cells incubated at $10^{\circ} \mathrm{C}$ for $2 \mathrm{~d}$ (Fig. 1E). These results suggested that temperature stress affects the expression of Grp94 that may regulate AChR clustering in cultured muscle cells.

To further investigate the regulation by HSP90 activity in aneural AChR clustering, we treated the cultured muscle cells with 17-AAG, a potent HSP90 inhibitor that alters the conformation of molecular chaperone machinery by inhibiting ATPase activity (Sharp and Workman, 2006). 17-AAG treatment exhibited a dose-dependent inhibition of aneural AChR cluster formation in cultured muscle cells (Fig. 1F). In cultured cells treated with 0.25 nм 17-AAG, some aneural AChR clusters were found, but they showed a significant reduction in the fluorescence intensity and topological complexity of perforated aneural AChR clusters (Fig. 1G,H), suggesting that HSP90 activity is involved in the formation and/or maintenance of topologically complex aneural AChR clusters. Taken together, these findings indicated that HSP90 expression and activity are precisely regulated at the optimal range to facilitate the formation of AChR clusters properly.

Previous studies showed that laminin-induced aneural AChR clusters in C2C12 myotubes can undergo topological transformation, mirroring the progressive morphologic changes in synaptic AChR clusters at NMJs in vivo (Kummer et al., 2004; Lee et al., 2009). We next examined whether HSP90 activity also participates in the topological remodeling of aneural AChR clusters. After identifying aneural AChR clusters in 2-d-old muscle cultures, timelapse imaging was then performed to monitor the dynamic changes in the morphology and intensity of the same AChR clusters in response to $1 \mathrm{~nm} 17-\mathrm{AAG}$ treatment over $48 \mathrm{~h}$. Specifically, preexisting and newly inserted AChRs were differentially labeled by BTX conjugated with red and green fluorophores, respectively, in accordance with a previously established protocol (Lee et al., 2009) and as illustrated in the schematic diagram (Fig. 1/). By examining the preexisting AChRs in control muscles, we observed a gradual dispersal of aneural AChR clusters, together with a reduction in AChR intensity, over a $48 \mathrm{~h}$ period (Fig. 1J,K). However, such spontaneous dispersal of aneural AChR clusters was greatly inhibited in the presence of 17-AAG. In contrast, the intensity of newly inserted AChRs between control and 17AAG-treated muscle cells showed no significant difference (Fig. 1J,L). Interestingly, instead of dispersing the entire aneural AChR clusters in control muscle cells, 17-AAG treatment caused a gradual loss of perforations, where podosome-like structures (PLSs) are located (Proszynski et al., 2009), within aneural AChR clusters (Fig. 1J, bottom panels, arrows). Considering the involvement of PLSs in AChR endocytosis and topological remodeling of aneural AChR clusters in Xenopus primary muscle cultures and $\mathrm{C} 2 \mathrm{C} 12$ myotubes (Lee et al., 2009; Proszynski et al., 2009; Yeo et al., 2015), we hypothesized that pharmacological inhibition of HSP90 activity affects the spatial localization of PLSs, leading to the stabilization of aneural AChR clusters. Collectively, our data demonstrated that HSP90 activity is involved in both the formation and topological remodeling/ dispersal of aneural AChR clusters in cultured muscle cells.

The HSP90 chaperone machinery is known to be a crucial regulator in maintaining cellular homeostasis under stressful conditions and normal metabolism (Schopf et al., 2017). To rule out the possibility that pharmacological inhibition of HSP90 activity by 17-AAG causes global metabolic changes in cultured muscle cells, we firstly performed GC-MS analysis to compare the levels of various polar metabolites and fatty acids between control and 17AAG-treated muscle cells. The PCAs showed that these 
two experimental groups from the same biological sample overlapped in the first principal component of polar metabolites or fatty acids (Extended Data Fig. 1-1A,C; Extended Data Table 1-1, Table 1-2). These results supported that 17-AAG treatment does not cause a significant change in the overall metabolite profile of cultured Xenopus muscle cells. Second, to eliminate the possible secondary effect of HSP90 inhibition on AChR clustering that is contributed by global changes in protein synthesis, we next used the OPP reagent, followed by click chemistry method to visualize the newly synthesized, nascent peptides/proteins (Liu et al., 2012; Slomnicki et al., 2016; Extended Data Fig. 1-1E). We detected a similar level of OPP signals between control and 17-AAG-treated muscle cells (Extended Data Fig. 1-1F). In contrast, $25 \mu \mathrm{M}$ CHX, a well-known protein synthesis inhibitor, showed a significant reduction in OPP signals. Third, to further validate the inhibitory effects on AChR clustering by 17-AAG are not because of defects in surface AChR insertion, we examined the density of single AChR molecules on the muscle surface using quantum dots as previously described (Geng et al., 2009; Extended Data Fig. 1-1G). We detected a similar density of surface AChR molecules between control and 17AAG-treated muscle cells (Extended Data Fig. 1-1H), indicating that de novo synthesis, followed by surface targeting mechanisms, of AChR proteins are not affected by HSP90 inhibition. Taken together, we provided multiple lines of evidence supporting that HSP90 inhibition causes a specific effect on AChR clustering and remodeling, rather than a global change of different cellular events in cultured muscle cells.

\section{Both diffuse and aneurally clustered AChRs contribute to the formation of synaptic AChR clusters}

Before synaptogenesis, AChR molecules are present in the surface of muscle fibers (preexisting AChRs) in the forms of aneurally clustered and diffuse receptors. It is believed that nerve innervation of skeletal muscles involves local signals to initiate synaptic AChR cluster formation and global signals to induce aneural AChR cluster dispersal (Dai and Peng, 1998). To demonstrate the differential contribution of aneurally clustered versus diffuse AChR molecules for the assembly of synaptic AChR clusters, laser-based photobleaching experiments were performed (Fig. 2). Specifically, all surface AChRs (diffuse and aneurally clustered ones, referred to as old AChR) were first labeled with Rh-BTX. In the experimental groups, a highpower laser was used to photobleach the fluorescence signals of aneural AChR clusters, while diffuse AChRs in the entire muscle cells were unaffected. Latex beads coated with recombinant agrin were then added onto muscle cells, which has previously been shown to induce AChR clustering at the bead-muscle contacts in a spatially and temporally controllable manner (Lee et al., 2009). After 1- or 3-d agrin bead stimulation, newly synthesized and inserted AChRs (referred to as new AChR) were labeled with 488-BTX. In the experimental group with photobleaching of aneural AChR cluster before agrin bead stimulation, we detected $52.66 \pm 0.05 \%$ reduction in the intensity of old AChR signals at $1-\mathrm{d}$ agrin bead- muscle contacts, compared with the control group without photobleaching (Fig. 2A,B). This result suggested that synaptic AChR clusters induced by agrin beads are recruited from both diffuse AChRs and aneural AChR clusters. Furthermore, the contribution of newly inserted AChRs to synaptic AChR clusters was negligible in the first day of agrin bead stimulation (Fig. 2A,C). Different from previous observations in cultured $\mathrm{C} 2 \mathrm{C} 12$ myotubes (Bruneau et al., 2008), our photobleaching approach however did not cause an effect similar to chromophore-assisted light inactivation (CALI) to induce dissipation of the illuminated aneural AChR clusters in cultured Xenopus muscle cells labeled with either 594-BTX or Rh-BTX (Extended Data Fig. 2-1). Therefore, our results indicated the differential contribution of aneurally clustered and diffuse AChRs for the assembly of synaptic AChR clusters, rather than the secondary effects caused by photo-dissipation of illuminated AChR clusters and their associated scaffolding proteins.

To further examine whether HSP90 regulates the recruitment of both diffuse AChRs and aneural AChR clusters to the nascent postsynaptic sites, the same laserbased photobleaching experiment was performed using muscle cells pretreated with $1 \mathrm{~nm} 17-\mathrm{AAG}$ for $1 \mathrm{~h}$ before the experiment. A similar level of old AChR signals was detected at agrin bead-muscle contacts in 17-AAGtreated muscle cells either with or without photobleaching the aneural AChR clusters, suggesting that 17-AAG treatment abolishes the recruitment of aneural AChR clusters, not diffuse AChRs, to the synaptic sites (Fig. 2A,B). On the other hand, the signals of new AChRs at agrin beadmuscle contacts showed no significant difference between control and 17-AAG-treated muscle cells (Fig. 2A, $C)$. As $\mathrm{HSP} 90 \beta$ plays a role in NMJ by regulating rapsyn localization and turnover (Luo et al., 2008), our immunostaining experiments further showed the association of rapsyn to those aneural AChR clusters that were stabilized by 17-AAG treatment in agrin bead-contacted muscles (Extended Data Fig. 2-2). In contrast, reduced rapsyn localization was detected at dispersing $\mathrm{AChR}$ clusters in control muscle cells after 4-8 $\mathrm{h}$ agrin bead stimulation. Taken together, these data suggested that aneural AChR clusters and diffuse AChRs contribute equally to the assembly of synaptic AChR clusters, and the recruitment of aneural AChR clusters, but not diffuse or newly inserted AChRs, to agrin-induced synaptic clusters is dependent on HSP90 activity and rapsyn turnover.

\section{Grp94 regulates ADF/cofilin-associated AChR redistribution on agrin stimulation}

To investigate whether Grp94 is the HSP90 isoform that regulates aneural AChR cluster formation, we used antisense $\mathrm{MO}$, which showed an effective knock-down of endogenous Grp94 expression level by Western blot analyses (Fig. $3 A, B$ ). Grp94 knock-down caused a significant inhibition in the formation of aneural AChR clusters, including both bottom and top clusters (Fig. 3D). Additionally, the intensity and complexity of aneural bottom AChR clusters were significantly reduced in Grp94 knock-down muscle cells (Fig. $3 C, E$ ), in agreement with 

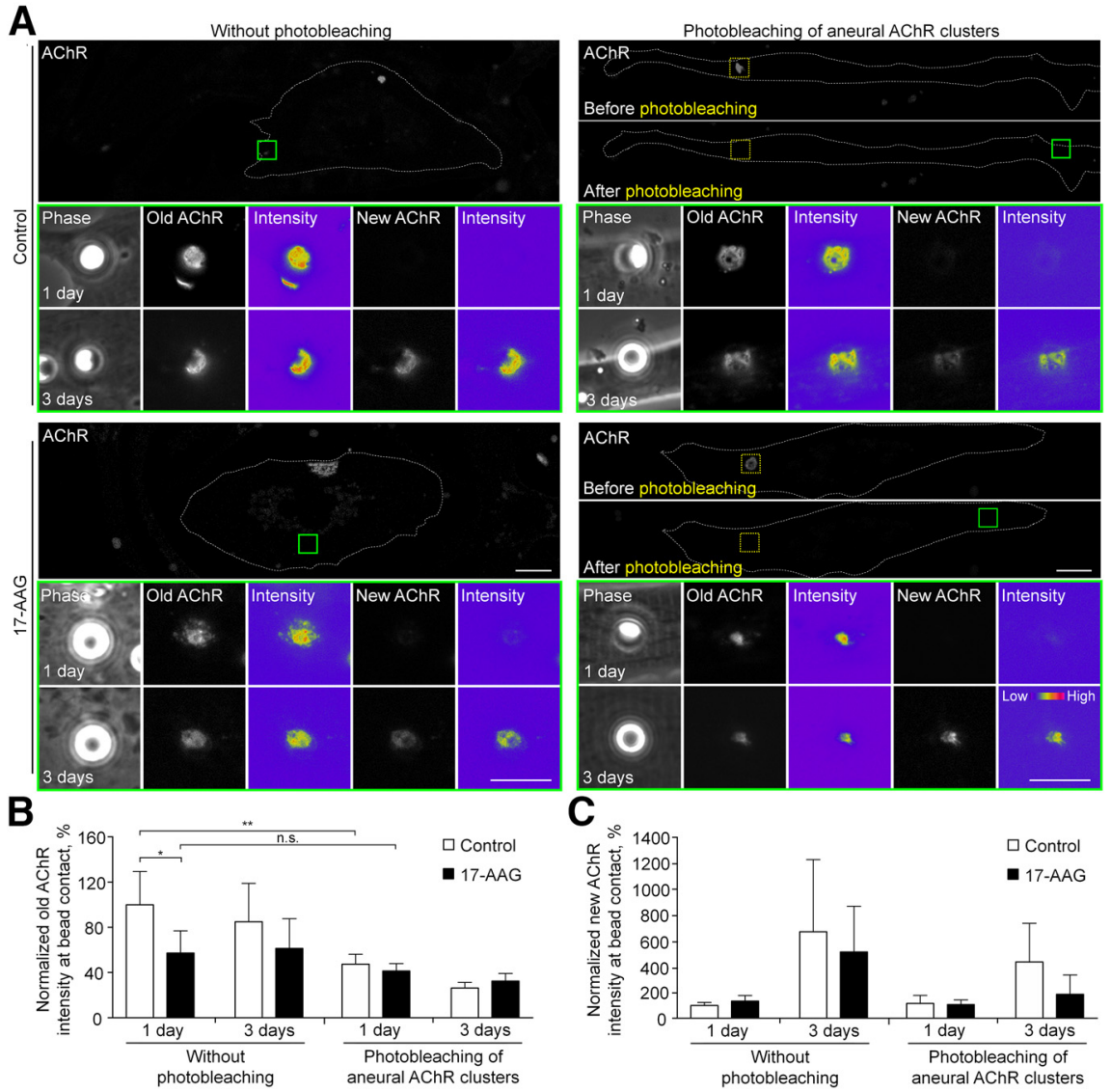

Figure 2. HSP90 regulates AChR recruitment from aneural clusters to agrin-induced clusters. $\boldsymbol{A}$, Representative images showing the differential contribution of diffuse and aneurally clustered AChRs to agrin bead-induced synaptic AChR clusters in control or 17AAG-treated muscle cells using laser-based photobleaching approach. Green boxes indicate the magnified view of muscle cells with agrin bead contacts at different time-points for clarity. Yellow dotted-line boxes indicate the photobleaching region of aneural AChR clusters before agrin bead stimulation. Dotted lines highlight the periphery of muscle cells. 8-bit pseudo-color images highlight the relative fluorescence intensity of preexisting (old) and newly inserted (new) AChR signals in muscle cells contacted by agrin beads for 1 and $3 \mathrm{~d}$. $\boldsymbol{B}, \boldsymbol{C}$, Quantification showing the fluorescence intensity of preexisting $(\boldsymbol{B})$ and newly inserted $(\boldsymbol{C})$ AChR signals at agrin bead-muscle contacts in control or 17-AAG-treated muscle cells, either with or without photobleaching of aneural AChR clusters before agrin bead stimulation; $n=11$ (control, without photobleaching), $n=16$ (control, photobleaching of aneural AChR clusters), $n=17$ (17-AAG-treated, without photobleaching), and $n=21$ (17-AAG-treated, photobleaching of aneural AChR clusters) muscle cells from three independent experiments. Scale bars: $10 \mu \mathrm{m}$. Data are shown as mean \pm SEM. Two-way ANOVA with Tukey's multiple comparisons test. $*$ and $* *$ represent $p \leq 0.05$ and 0.01 , respectively. n.s.: non-significant.

the observations in 17-AAG-treated muscles (Fig. 1G). Given the known function of HSP90 $\beta$ in agrin-induced AChR cluster formation and maintenance (Luo et al., 2008), we here showed that another HSP90 protein Grp94 is required for the initial formation of aneural AChR clusters.

A previous study demonstrated that ADF/cofilin localization is spatiotemporally correlated with AChR redistribution from aneural to agrin-induced clusters (Lee et al., 2009). We next further studied whether Grp94 is required for ADF/cofilin-mediated AChR redistribution. To test this, we performed dual-channel time-lapse imaging to monitor the change of AChR cluster morphology and intensity in correlation with ADF/cofilin localization at aneural AChR clusters in the same muscle cells before and after agrin bead stimulation (Fig. $3 F-/$ ). Consistent with previous studies (Lee et al., 2009), we also observed spatially localized GFP-XAC to be associated with agrin-induced
AChR clusters at the bead-muscle contacts, which was accompanied with the reduction of localized GFP-XAC signals at the dispersing aneural AChR clusters, in muscle cells with GFP-XAC alone or with Control MO + GFPXAC. However, in Grp94 MO muscle cells, agrin-induced AChR clusters at the bead-contacted sites were significantly reduced, and the morphology and intensity of aneural AChR clusters remained largely unchanged before and after agrin bead stimulation (Fig. 3F-l). Importantly, the spatial localization of GFP-XAC associated with aneural and agrin-induced AChR clusters was largely reduced in Grp94 knock-down muscles (Fig. 3F,H,I). Taken together, our data suggested that Grp94 is required for the formation and dispersal of agrin-induced and aneural AChR clusters, respectively, possibly through modulating the spatial localization of ADF/cofilin.

Since ADF/cofilin is also considered as one of the PLS core markers to be associated with AChR clusters (Chan 


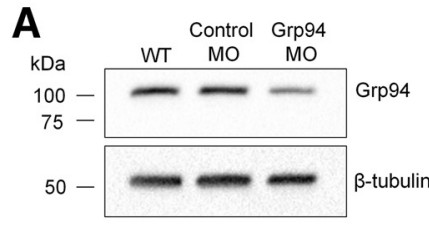

B

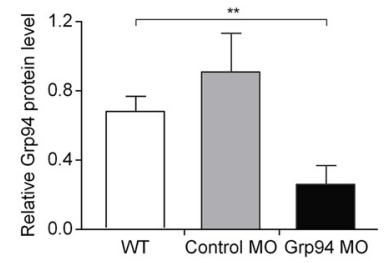

$\mathbf{F}$

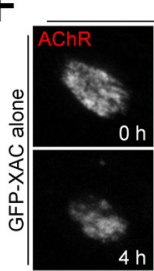

Aneural AChR clusters
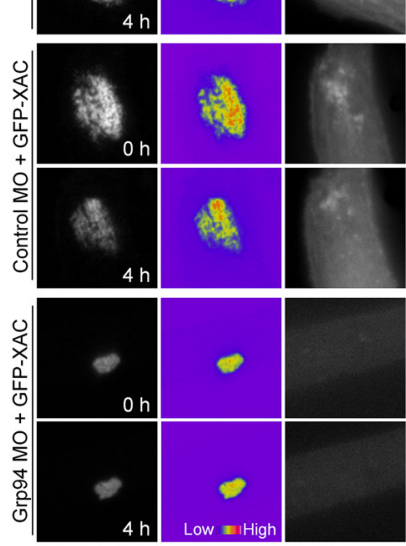
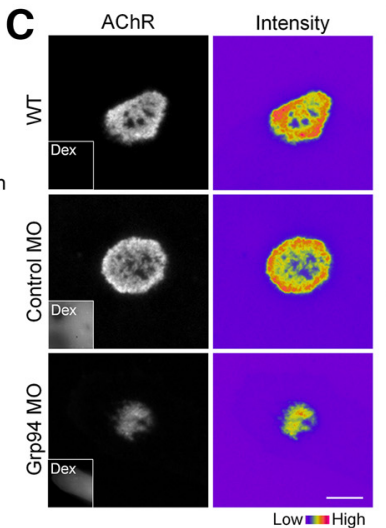

E
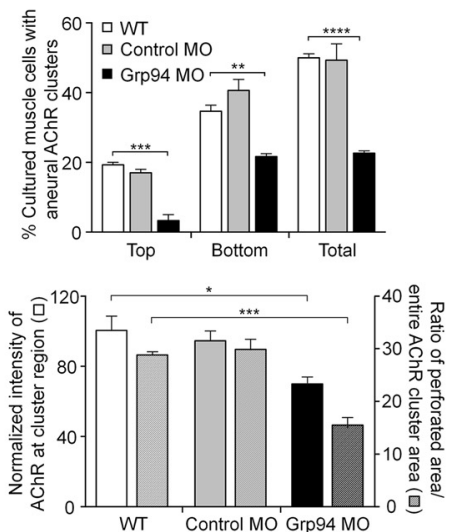

G

$4 \mathrm{~h}$ bead-muscle contacts
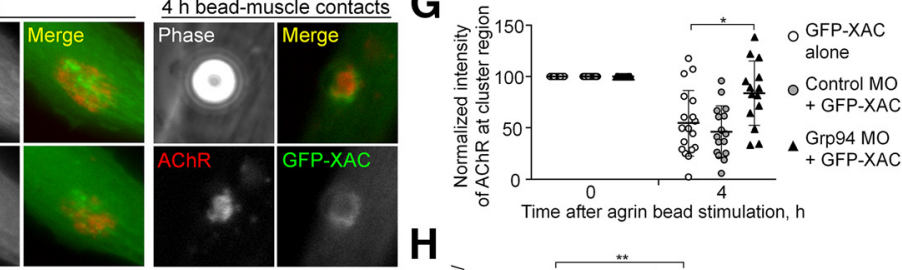

$\mathrm{H}$
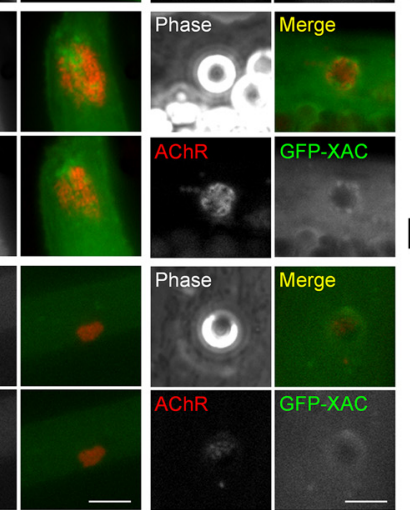

Figure 3. Grp94 knock-down inhibits agrin bead-induced AChR clustering by modulating ADF/cofilin localization. $\boldsymbol{A}$, $\boldsymbol{B}$, Western blot analysis $(\boldsymbol{A})$ and quantification $(\boldsymbol{B})$ showing the expression level of Grp94 in WT, Control MO, and Grp94 MO embryos. $\beta$-Tubulin was used as the loading control for normalization. $\boldsymbol{C}$, Representative images showing the intensity and complexity of aneural AChR clusters in response to Grp94 knock-down. 8-bit pseudo-color images highlight the relative fluorescence intensity of AChR clusters in control versus Grp94 knock-down muscle cells. Insets show the fluorescent dextran (Dex) signals, indicating the presence of MO. D, Quantification showing the effects of MO-mediated Grp94 knock-down on the formation of aneural AChR clusters in cultured muscles; $n=150$ (WT), $n=143$ (Control MO), and $n=150$ (Grp94 MO) muscle cells from three independent experiments. $\boldsymbol{E}$, Quantification showing the effects of MO-mediated Grp94 knock-down on the intensity and complexity of aneural AChR clusters; $n=64$ (WT), $n=47$ (Control MO), and $n=52$ (Grp94 MO) muscle cells from four independent experiments for fluorescence intensity measurement (left $y$-axis); $n=44$ (WT), $n=42$ (Control MO), and $n=44$ (Grp94 MO) muscle cells from four independent experiments for cluster complexity measurement (right $y$-axis). $\boldsymbol{F}$, Representative sets of time-lapse images showing the effects of Grp94 knock-down on GFP-XAC localization in association with the dispersal of aneural AChR clusters (left panels) and with the formation of agrin bead-induced AChR clusters (right panels). 8-bit pseudo-color images highlight the change in fluorescence intensity of aneural AChR clusters after agrin bead stimulation for $4 \mathrm{~h}$. $\mathbf{G}, \boldsymbol{H}$, Individual value plots showing the percentage change in the fluorescence intensities of AChRs $(\boldsymbol{G})$ and GFP-XAC $(\boldsymbol{H})$ in the same aneural AChR clusters among different conditions after agrin bead stimulation for $4 \mathrm{~h} ; n=12$ (GFP-XAC alone), $n=9$ (Control MO + GFP-XAC), and $n=11$ (Grp94 MO + GFP-XAC) muscle cells from three independent experiments. I, Quantification showing the effects of Grp94 knock-down on agrin bead-induced AChR clustering (left $y$-axis) and GFP-XAC localization (right $y$-axis); $n=24$ (GFP-XAC alone), $n=24$ (Control MO + GFP-XAC), and $n=21$ (Grp94 MO + GFP-XAC) muscle cells from four independent experiments. Scale bars: $5 \mu \mathrm{m}$. Data are shown as mean \pm SEM (B, $\boldsymbol{D}, \boldsymbol{E}, \boldsymbol{I})$ or mean $\pm \mathrm{SD}(\boldsymbol{G}, \boldsymbol{H})$. One-way ANOVA with Dunnett's multiple comparisons test $(\boldsymbol{B}, \boldsymbol{E})$, two-way ANOVA with Tukey's multiple comparisons test $(\boldsymbol{D})$, two-way ANOVA with Sidak's multiple comparison test $(\boldsymbol{G}, \boldsymbol{H})$ and one-way ANOVA with Turkey's multiple comparison test $(\boldsymbol{I})$.*,**,***, and $* * * *$ represent $p \leq 0.05,0.01,0.001$, and 0.0001 , respectively. n.s.: non-significant. 


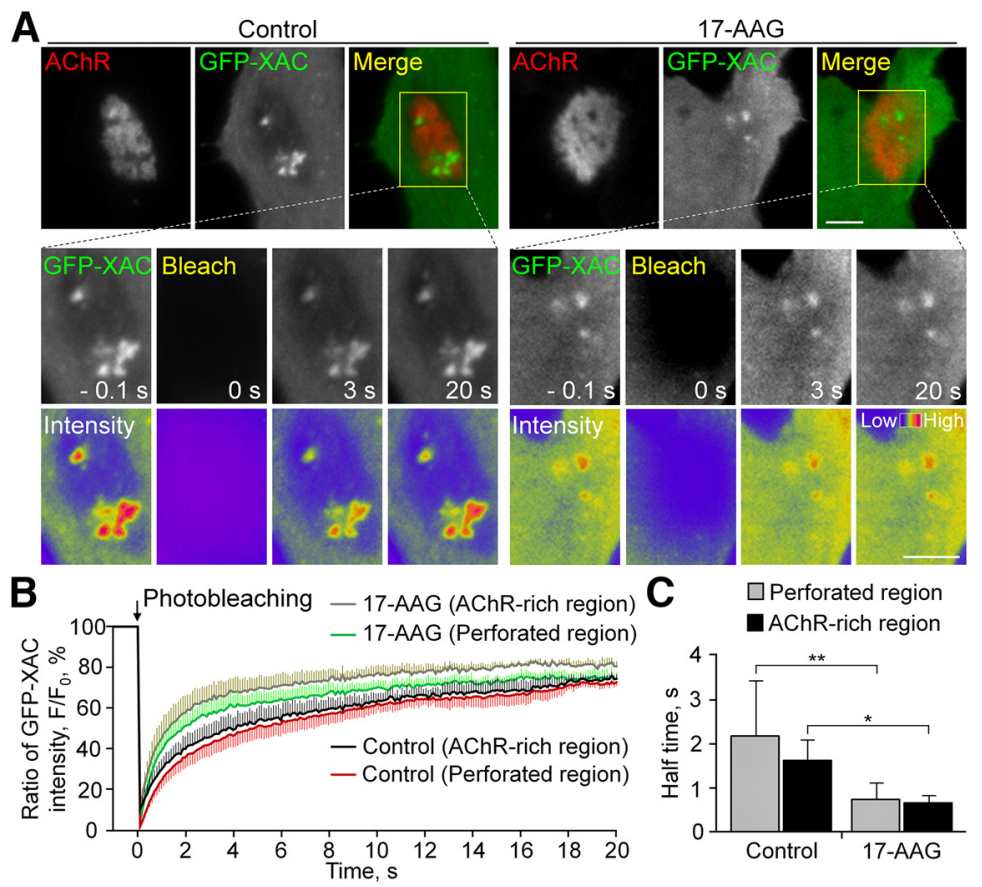

Figure 4. HSP90 inhibition accelerates ADF/cofilin turnover at different regions of aneural AChR clusters. A, Representative timelapse TIRF images showing the fluorescence recovery of GFP-XAC signals after photobleaching the region of aneural AChR clusters (yellow rectangles, which are magnified in bottom rows with multiple timepoints) in control or 17-AAG-treated muscle cells. 8-bit pseudo-color images highlight the relative fluorescence intensity of GFP-XAC signals. $\boldsymbol{B}, \boldsymbol{C}$, Quantification showing the FRAP curves $(\boldsymbol{B})$ and the calculated recovery half-time $(\boldsymbol{C})$ of GFP-XAC signals at perforated and AChR-rich regions within aneural AChR clusters in control versus 17-AAG-treated muscle cells; $n=12$ (Control) and $n=7$ (17-AAG) muscle cells from three independent experiments. Scale bars: $5 \mu \mathrm{m}$. Data are shown as mean \pm SEM. Student's $t$ test. $*$ and $* *$ represent $p \leq 0.05$ and 0.01 , respectively.

et al., 2020b), the reduced complexity of aneural AChR clusters in Grp94 knock-down muscle cells may be because of the dysregulation of PLS localization. To determine the requirement of Grp94 for the spatial localization of PLSs at aneural AChR clusters, our immunostaining experiments showed that XAC and p34-Arc, one of the Arp2/3 subunits, were spatially localized at the perforations of aneural AChR clusters in WT or Control MO muscles, but not in Grp94 MO muscles (Extended Data Fig. 3-1A-C). Likewise, PLS cortex marker vinculin was found to be localized at the edge of perforations within AChR clusters and at the cell periphery of WT or Control MO muscles, but its spatial localization patterns were significantly reduced in Grp94 MO muscles (Extended Data Fig. 3-1A,D). These results suggested that MO-mediated knock-down of endogenous Grp94 may also affect proper localization of PLSs at aneural AChR clusters, which in turn affect the stability of aneural AChR clusters and their contribution to the synaptic clusters under synaptogenic stimulation.

\section{Grp94 regulates spatial localization and dynamic turnover of ADF/cofilin at aneural AChR clusters}

Since spatially localized ADF/cofilin may direct AChR endocytosis, trafficking, and/or insertion for the assembly of synaptic AChR clusters at developing NMJs via transcytosis (Lee et al., 2009; Yeo et al., 2015), we hypothesized that Grp94 activity regulates the dynamic turnover of localized ADF/cofilin so as to control the stability of aneural AChR clusters. To test this, we performed TIRF imaging and FRAP experiments on GFP-XAC-overexpressing muscle cells either with or without 17-AAG treatment (Fig. 4). Specifically, laser-based photobleaching was conducted at the region of aneural AChR clusters (Fig. $4 A$, yellow rectangles), followed by time-lapse imaging to monitor the recovery of GFP-XAC fluorescence signals in the photobleached region (Fig. $4 A, B$ ). In control muscle cells, we detected a rapid recovery (in seconds after photobleaching) of GFP-XAC signals at aneural AChR clusters. By examining the half-time of fluorescence recovery (the time taken for the fluorescence intensity to recover to half of the plateau level), we found that 17-AAG treatment caused a significant reduction in the recovery half-time of GFP-XAC signals by $59 \%$ to $0.86 \pm 0.2 \mathrm{~s}$ in the perforated regions of AChR clusters (Fig. $4 C$ ), indicating a much faster turnover rate of ADF/ cofilin at aneural AChR clusters on HSP90 inhibition. Interestingly, we also detected a significant reduction in the recovery half-time of GFP-XAC in the AChR-rich region of aneural clusters by 17-AAG treatment, suggesting that HSP90 inhibition increases the turnover of perimembrane fraction of ADF/cofilin at not only the perforated regions, but also the AChR-rich regions, of aneural clusters.

In migrating cells, HSP90 is known to form a molecular complex containing slingshot (SSH), a serine/threonine phosphatase that dephosphorylates and activates ADF/ cofilin, to regulate lamellipodial protrusion and directed motility (Fotedar and Margolis, 2015), indicating that 
HSP90 may modulate the phosphorylation state of ADF/ cofilin. To test whether HSP90-regulated ADF/cofilin turnover is mediated through phosphocycling, we performed TIRF-FRAP experiments to compare the fluorescence recovery rate in muscle cells overexpressing different phosphorylation mutant or WT forms of GFP-XAC (Extended Data Fig. 4-1). The constitutively active (S3A) mutant of GFP-XAC exhibited a much slower recovery half-time than the WT form at both perforated and AChR-rich regions of aneural clusters, suggesting that active ADF/cofilin molecules bind to and modulate the dynamic turnover of PLS and cortical actin at perforated and AChR-rich regions of AChR clusters, respectively. Similar to the effects of 17-AAG treatment (Fig. 4C), the inactive ADF/cofilin mutant (S3E) showed a significant reduction in the recovery half-time at both perforated and AChR-rich regions of aneural clusters, compared with the WT form. These data suggested that HSP90 may modulate ADF/cofilin activity via phosphorylation at its serine- 3 residue.

\section{Postsynaptic Grp94 regulates synaptic structure and function of developing NMJs}

To examine the roles of postsynaptic Grp94 in the formation of NMJs in vitro, we examined nerve-induced AChR clustering in Xenopus nerve-muscle co-cultures by knocking down the endogenous expression of muscle Grp94 specifically. In WT nerve-muscle co-cultures [WT $(\mathrm{M}+\mathrm{N})$ ] or in the chimeric co-cultures containing Control $\mathrm{MO}$ muscles and WT neurons [Control MO $(\mathrm{M})+\mathrm{WT}(\mathrm{N})$ ], nerve-induced synaptic AChR clusters were highly concentrated along the nerve-muscle contact sites (Fig. 5A). In contrast, we observed a significant reduction in the percentage of nerve-muscle contacts with AChR clusters and the fluorescence intensity of nerve-induced AChR clusters in the chimeric co-cultures containing Grp94 MO muscles and WT neurons [Grp94 MO (M) + WT (N)] (Fig. $5 A-C)$. These data suggested that Grp94 is required for the formation of nerve-induced AChR clusters, consistent with the requirement of Grp94 for agrin bead-induced AChR clustering as shown above (Fig. 3F). To determine whether pharmacological inhibition of HSP9O or molecular manipulation of Grp94 expression affects the spatial localization of rapsyn that leads to the reduced density of synaptic AChR clusters, we performed rapsyn immunostaining in nerve-muscle co-cultures treated with different HSP90 inhibitors or in different chimeric co-cultures (Extended Data Fig. 5-1). In WT [WT (M + N)] or in the chimeric co-cultures containing Control $\mathrm{MO}$ muscles and WT neurons [Control MO (M) + WT (N)], rapsyn was spatially colocalized with nerve-induced synaptic AChR clusters (Extended Data Fig. 5-1A). However, significant reductions in AChR intensity and its associated rapsyn signals were detected in co-cultures treated with 17-AAG or PU-WS13, a specific Grp94 inhibitor. Similar observations were made in the chimeric co-cultures containing Grp94 MO muscles and WT neurons [Grp94 MO (M) + WT (N)] (Extended Data Fig. 5-1), indicating muscle Grp94 regulates the assembly of nerve-induced synaptic AChR clusters by modulating the precise localization of postsynaptic scaffold protein rapsyn.
To further understand the role of HSP90 or Grp94 in $A C h R$ redistribution from aneural to synaptic AChR clusters, we next examined the amount of internalized $A C h R$ vesicles at aneural AChR clusters on agrin bead stimulation in the presence of 17-AAG or PU-WS13 (Extended Data Fig. 5-2A). Compared with control cells, HSP90 or Grp94 inhibition significantly reduced the number of internalized AChR vesicles at aneural AChR clusters in agrin bead-contacted muscle cells (Extended Data Figs. 5-2A,B). Taken together, our findings suggested that Grp94 is the specific HSP90 family member that regulates the redistribution and recruitment of aneural AChR clusters to the postsynaptic specializations, likely through ADF/cofilin-mediated transcytosis mechanism as previously proposed (Lee et al., 2009).

To determine the effects of postsynaptic Grp94 knockdown on synaptic functions of developing NMJs, we performed whole-cell voltage-clamp recordings to examine the SSCs in 1-d-old Xenopus nerve-muscle co-cultures (Fig. 5D). In the chimeric co-cultures of Grp94 MO (M) + WT $(\mathrm{N})$, we detected a significant reduction in the amplitude of SSCs (Fig. 5E,F), which is likely attributed by the reduced $A C h R$ density at the nerve-muscle contacts as observed in our cell imaging studies (Fig. 5A). On the other hand, we also detected a significant reduction in SSC frequency, leading to a right shift pattern in the cumulative distribution of inter-event intervals (Fig. 5G,H). In contrast, postsynaptic Grp94 knock-down caused no significant effects on the rise time and decay time of SSCs (Fig. $5 /, J$ ), demonstrating that the channel properties of AChRs in muscles remain unaffected. In summary, this study identified postsynaptic Grp94 as a novel regulator to control the synaptic structures and functions at developing NMJs.

\section{Discussion}

Temperature has long been considered as a physiological factor that affects neuronal growth and maintains synaptic homeostasis by modulating presynaptic and postsynaptic elements at the invertebrate NMJs (Tsai et al., 2012; Yeates et al., 2017; Zhu et al., 2018). South African clawed toad, Xenopus laevis, is an ectotherm vertebrate that has been widely used as an excellent animal model for embryology studies (Hobson, 1965). Since the body temperature of Xenopus is subjected to fluctuations in environmental temperature, this provides an ideal model for studying the molecular mechanisms underlying temperature-dependent alterations in the structure and functions of the vertebrate NMJs. In this study, we provided evidence showing that temperature stress-modulated Grp94 expression and activity regulate the recruitment of aneural AChR clusters for the assembly of postsynaptic specializations through modulating ADF/cofilin activity, suggesting a novel role of Grp94 in the formation of vertebrate NMJs. Consistent with a previous study showing the function of cytosolic HSP90 member, $\operatorname{HSP} 90 \beta$, for regulating rapsyn turnover and agrin-induced $A C h R$ cluster formation (Luo et al., 2008), our present study further identified another HSP90 family member Grp94, an ER-resident molecular chaperone, in regulating AChR clustering and remodeling during NMJ development. 
A
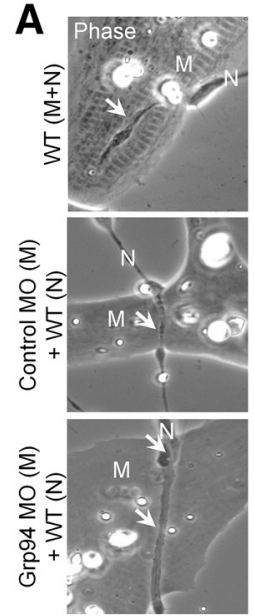

D

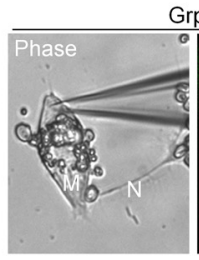

E

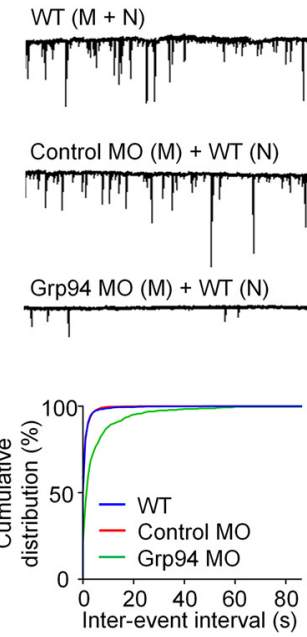

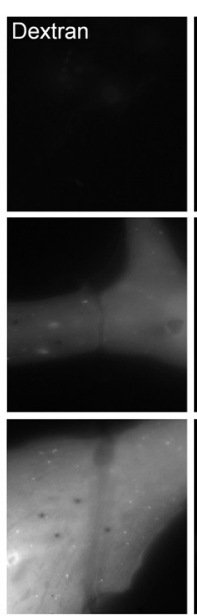

Grp94 MO (M) + WT (N)
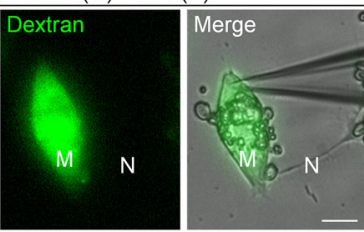

G

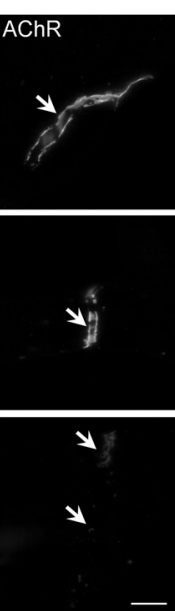

B

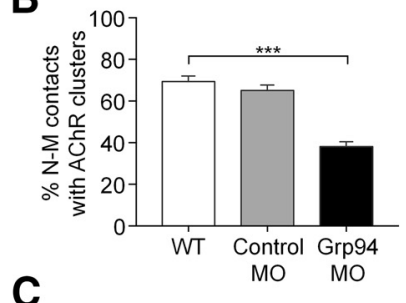

C

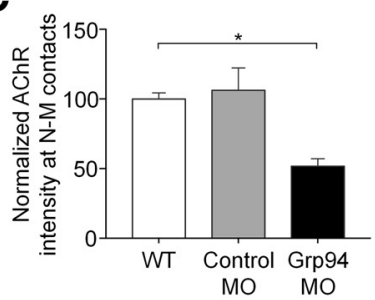

F
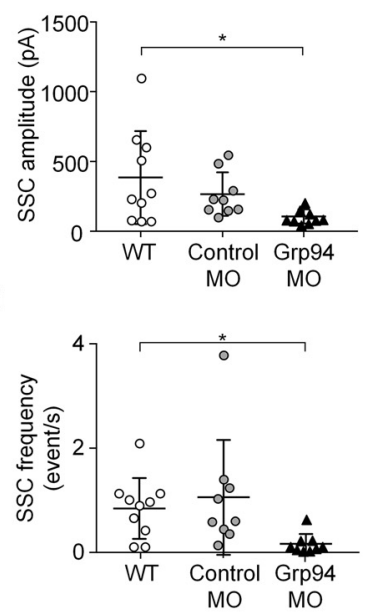

J

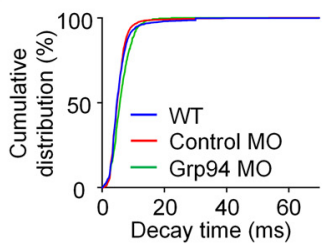

Figure 5. Postsynaptic Grp94 knock-down impairs synaptic structures and functions at developing NMJs. A, Representative images showing the effects of muscle Grp94 knock-down on nerve-induced AChR clustering. Fluorescent dextran signals indicate the presence of MO. Arrows indicate sites of nerve-muscle contacts. B, C, Quantification showing the effects of muscle Grp94 knock-down on the percentage of nerve-muscle contacts with AChR clusters $(\boldsymbol{B})$ and the fluorescence intensity of nerve-induced AChR clusters (C) in 1-d-old Xenopus nerve-muscle co-cultures; $n=150$ (WT), $n=146$ (Control MO), and $n=191$ (Grp94 MO) nerve-muscle contacts from three independent experiments for quantifying the percentage of nerve-muscle contacts with synaptic AChR clusters (B); $n=33$ (WT), $n=31$ (Control MO), and $n=33$ (Grp94 MO) nerve-muscle contacts from three independent experiments for measuring $A C h R$ fluorescence intensity $(\boldsymbol{C})$. $\boldsymbol{D}$, Representative images showing the whole-cell patch-clamp recording on a Grp94 MO muscle cell innervated by a WT spinal neuron. Fluorescent dextran signals indicate the presence of MO. E, Representative electrophysiological recording traces of SSCs recorded from WT, Control MO, or Grp94 MO muscles that were innervated by WT spinal neurons. $\boldsymbol{F}, \mathbf{G}$, Quantification showing the effects of muscle Grp94 knock-down on the amplitude $(\boldsymbol{F})$ and frequency $(\boldsymbol{G})$ of SSCs. $\boldsymbol{H}-\boldsymbol{J}$, Cumulative distribution plots of the inter-event interval $(\boldsymbol{H}), 10-90 \%$ rise time $(\boldsymbol{I})$, and decay time $(\boldsymbol{J})$ of SSCs recorded from WT, Control MO, or Grp94 MO muscles innervated by WT spinal neurons. $n=10$ (WT), $n=9$ (Control MO), and $n=9$ (Grp94 MO) nerve-muscle pairs from three independent experiments (F-J). Scale bars: $10 \mu \mathrm{m}$. "M": muscle; "N": neuron. Data are represented as mean $\pm \operatorname{SEM}(\boldsymbol{B}, \boldsymbol{C})$ and mean \pm SD $(\boldsymbol{F}, \boldsymbol{G})$. One-way ANOVA with Tukey's multiple comparisons test $(\boldsymbol{B}, \boldsymbol{C})$. Kruskal-Wallis ANOVA test with Dunn's multiple comparison test $(\boldsymbol{F}, \boldsymbol{G})$. $*$ and $* * *$ represent $p \leq 0.05$ and 0.001 , respectively. 
HSP90 proteins are implicated in diverse biological processes, in which a variety of coordinated regulatory mechanisms are involved to control their expression and activity (Prodromou, 2016). In response to stressful conditions, heat shock factor 1 is an important regulator responsible for the transcriptional regulation of HSP90 genes. In this study, mRNA levels of HSP90 $\beta$ and Grp94 were upregulated in cultured Xenopus muscle cells by low-temperature treatment (Fig. 1C). However, Grp94 protein level was found to be significantly reduced under low-temperature stress (Fig. 1D,E). This discrepancy between mRNA and protein levels of Grp94 could be explained by a negative feedback mechanism (DiDomenico et al., 1982; Bader et al., 2015), in which Grp94 mRNA may be upregulated to compensate a reduced amount of Grp94 protein level. As both temperature stress and HSP90 inhibitor significantly suppress the formation of aneural AChR clusters, this suggests that HSP90 expression and activity are essential for the regulation of AChR clustering in cultured muscle cells.

One common concern with all HSP90 studies is that pharmacological inhibition of HSP90 activity may be causing non-specific, global changes in cell metabolism and protein expression. Here, we employed multiple experimental approaches to show that it is unlikely the case in the present study: targeted metabolomics (polar metabolites and fatty acids) study (Extended Data Figs. 1-1A-D), nascent protein synthesis assay (Extended Data Fig. 1-1E,F), single AChR molecule labeling (Extended Data Fig. 1-1G,H), and newly inserted AChR labeling (Fig. 2C). Together, results from all these experiments indicated that HSP90 inhibition by $17-A A G$ causes a specific effect on AChR clustering and remodeling, rather than a plethora of changes in different cellular events.

Previous genetic studies demonstrated that nerve-independent formation of AChR prepatterns can be detected in the central region of muscle fibers at developing NMJs in vivo (Yang et al., 2000, 2001; Lin et al., 2001). Similarly, spontaneously formed aneural AChR clusters can be found in cultured muscle cells, suggesting muscle-intrinsic mechanisms underlying initial AChR cluster formation. Upon synaptogenic induction, it is hypothesized that the dispersal of aneural AChR clusters is temporally coupled with the formation of synaptic AChR clusters (Dai and Peng, 1998). A previous study performed by single AChR tracking approach showed the contribution of surface AChR molecules, derived from either aneural clusters or diffuse receptor pool, to the sites of nerve-muscle contacts (Geng et al., 2009). Interestingly, a recent study further provided a definite evidence to demonstrate the recruitment of aneural AChR clusters for the assembly of nerve-induced synaptic AChR clusters (Chan et al., 2020b). Consistent with that, our present study confirmed the differential contribution of preexisting AChRs (from aneural clusters and diffuse AChRs) versus newly synthesized and inserted AChRs for the assembly of agrin-induced postsynaptic specializations (Fig. 2), in which HSP90 or Grp94 is required for the recruitment of AChRs from aneural clusters, but not from diffuse nor newly inserted ones. The stability of AChR clusters is known to be regulated by rapsyn, a multidomain synaptic adaptor protein for AChR anchoring by interacting directly with actin or indirectly with various cytoskeletal regulatory proteins (Wang et al., 1999; Oury et al., 2019; Xing et al., 2020). Apart from serving as a scaffolding molecule at NMJs, rapsyn contains E3 ligase activity that increases neddylation of AChR subunits (Li et al., 2016), leading to the stabilization of AChR clusters. Consistent with this notion, we observed the association of rapsyn with aneural AChR clusters that are stabilized by HSP90 inhibition (Extended Data Fig. 2-2), preventing AChR molecules of aneural clusters from being recruited to the synaptic sites.

It is worth noting that our laser-based photobleaching experiments did not cause photo-dissipation of illuminated AChR clusters and their intracellular scaffolding proteins (Extended Data Fig. 2-1), as previously observed in cultured C2C12 myotubes (Bruneau et al., 2008). Although the exact molecular mechanism underlying photo-dissipation of AChR clusters remains unknown, illumination of a photosensitizer chromophore (e.g., Alexa Fluor 594) may generate reactive oxygen species, leading to limited damage of surrounding target proteins through CALI. In early Xenopus embryos, there are various genetically regulated enzymes involved in antioxidant defences (Rizzo et al., 2007). These enzymes may dampen the possible effects of photo-dissipation in cultured Xenopus muscle cells. Therefore, our experimental approach allows us to study the essential roles of AChR molecules derived from dispersing aneural AChR clusters, rather than the destruction and removal of illuminated AChR clusters and their associated scaffolding proteins, in the assembly of agrin-induced synaptic AChR clusters.

PLS is composed of a core domain containing F-actin and its associated proteins such as ADF/cofilin, Arp2/3 complex, and cortactin, as well as a cortex domain containing focal adhesion proteins such as talin, vinculin, and paxillin. Apart from actin-binding proteins, a recent study also showed that a microtubule-binding protein, microtubule-actin cross linking factor 1 (Macf1), is concentrated at PLS within AChR clusters (Oury et al., 2019). Among different PLS proteins, ADF/cofilin is known to regulate AChR endocytosis, trafficking, and/or insertion at developing NMJs (Lee et al., 2009; Yeo et al., 2015). The actin-binding activity of ADF/cofilin is tightly controlled by a balancing act of phosphorylation and dephosphorylation on its serine3 residue by LIM or testicular (TES) kinases and SSH phosphatase, respectively. Active, non-phosphorylated ADF/cofilin mediates actin depolymerization by binding to and severing F-actin, while serine-3 phosphorylated ADF/cofilin inhibits its binding to G-actin and F-actin (Bernstein and Bamburg, 2010). As a previous study indicated, constitutively active (S3A) mutant of GFP-XAC is highly localized at the perforated regions of aneural AChR clusters compared with inactive (S3E) mutant or WT forms of GFP-XAC (Lee et al., 2009), suggesting that active ADF/cofilin molecules are preferentially localized at perforated regions of AChR clusters to spatially modulate actin dynamics. Surprisingly, we found that 17-AAG treatment caused a significant increase 


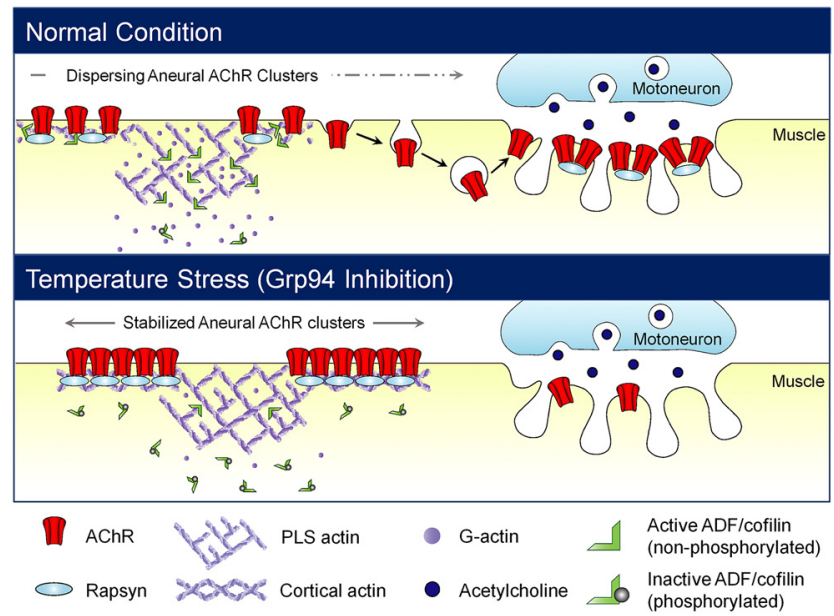

Figure 6. Temperature stress-induced Grp94 inhibition affects AChR recruitment from aneural to synaptic clusters by modulating ADF/cofilin phosphorylation and activity. To allow AChR redistribution during neuromuscular synaptogenesis, modulation of actin dynamics at the cell cortex and at the PLS are required for mobilizing AChR molecules and facilitating vesicular trafficking of AChR molecules at aneural clusters, respectively. Our findings suggest that temperature stress-induced Grp94 inhibition promotes phosphorylation or suppress dephosphorylation of ADF/cofilin at perforated and AChR-rich regions of aneural clusters, thereby stabilizing them against agrin-induced dispersal and recruitment to the postsynaptic sites.

in the turnover rate of GFP-XAC at both perforated and AChR-rich regions of aneural clusters (Fig. 4), suggesting that ADF/cofilin modulates actin dynamics at not only the PLS, but also the cell cortex in association with AChR molecules. Cortical actin filaments are organized as a dense meshwork that lies directly underneath the plasma membrane (Chugh and Paluch, 2018), where ADF/cofilin-mediated dynamics of cortical actin filaments may facilitate endocytosis and exocytosis events in vesicular trafficking of AChR molecules (Lee et al., 2009, 2014). Similar to the effects of HSP90 inhibition, we observed an increased turnover rate in muscle cells expressing phospho-mimic inactive S3E mutant of GFP-XAC (Extended Data Fig. 4-1), suggesting that HSP90 inhibition may promote phosphorylation or suppress dephosphorylation of ADF/cofilin at perforated and AChR-rich regions of aneural clusters. The phosphorylated $A D F / c o f i l i n$ is inactive in actin binding, therefore it is incapable of modulating the dynamics of both cortical actin (for mobilizing AChR molecules) and PLS actin (for directing vesicular trafficking of AChR molecules) at aneural clusters (Fig. 6).

As HSP90 is known to form a molecular complex containing SSH in migrating cells (Fotedar and Margolis, 2015), HSP90 may indirectly modulate the phosphorylation state and turnover rate of ADF/cofilin in cultured muscle cells. Apart from the phosphorylation regulation, a recent study has identified that cofilin is a physiologically relevant neddylation target that modulates cytoskeletal actin dynamics in neuronal outgrowth (Vogl et al., 2020). Since rapsyn contains E3 ligase activity (Li et al., 2016), it would be of interest to determine whether rapsyn regulates the dynamic turnover of ADF/cofilin via neddylation at AChR clusters. In addition, the spatial localization of ADF/cofilin at the postsynaptic sites can also be regulated by 14-3-3 $\zeta$ (Lee et al., 2009). As ER stress inducers are known to modulate the expression levels of 14-3-3 $\zeta$ and Grp94 in hippocampal neurons (Murphy et al., 2008; Brennan et al., 2013), whether temperature stress-induced alterations in Grp94 expression and activity affect $14-3-3 \zeta$-regulated ADF/cofilin localization at AChR clusters remain to be examined. Taken together, we speculate that Grp94 inhibition or knock-down may affect either the dynamic turnover (via phosphocycling and/or neddylation) or the spatial localization (via 14-3$3 \zeta)$ of ADF/cofilin, which in turn controls actin-mediated vesicular trafficking of AChRs in the formation of NMJs. Future studies will be focused on elucidating the detailed molecular mechanisms underlying how Grp94 regulates the upstream regulators of ADF/cofilin activity and localization in aneural versus synaptic AChR clusters at developing NMJs.

In the electrophysiological recordings, we showed a significant reduction of SSC frequency in the chimeric co-cultures of Grp94 knock-down muscles and WT neurons (Fig. 5G). This effect may be attributed by the reduced probability of neurotransmitter release, suggesting that postsynaptic Grp94 may also be involved in retrograde signaling to affect presynaptic functions. As Grp94 is one of the major $\mathrm{Ca}^{2+}$ binding proteins at the ER (Van et al., 1989), it has to contend during the fluctuations in free $\mathrm{Ca}^{2+}$ in the lumen, as protein-bound $\mathrm{Ca}^{2+}$ is released through the ER membrane channels to the cytosol in response to the physiological demands of the cell (Marzec et al., 2012). Previous studies identified that the activity of $\mathrm{Ca}^{2+}$ /calmodulin-dependent protein kinase II (CaMKII) in postsynaptic muscles retrogradely modulates the neurotransmitter release at Drosophila NMJs (Haghighi et al., 2003). Additionally, conditional deletion of Grp94 results in the loss of $\beta$-catenin signaling in the intestinal epithelium (Liu et al., 2013). While muscle $\beta$-catenin is known to retrogradely regulate presynaptic differentiation and function at NMJs ( $\mathrm{Li}$ et al., 2008), it will be of interest to investigate whether postsynaptic Grp94 serves as a regulator of CaMKIImediated or $\beta$-catenin-mediated retrograde signaling to affect presynaptic structure and function.

In summary, this study provides the first evidence suggesting that temperature stress regulates the development of vertebrate NMJs through the expression and activity of postsynaptic Grp94. It is important to note that auto-antibodies against Grp94 has recently been identified in myasthenia gravis (MG), an autoimmune NMJ disease (Suzuki et al., 2011). Another recent study has also shown a significant positive correlation between the age of MG onset and the expression level of Grp78, another ER chaperone (Iwasa et al., 2014). Therefore, results of our study provide insights into not only the fundamental mechanisms underlying the vertebrate NMJ development, but also the pathogenic mechanisms underlying ER stress response and NMJ disruption in MG. 
Table 1: Summary of statistical analyses

\begin{tabular}{|c|c|c|c|c|c|}
\hline \multirow{2}{*}{$\frac{\text { Figures }}{1 B}$} & \multicolumn{2}{|l|}{ Comparison } & \multirow{2}{*}{$\begin{array}{l}\text { Statistical test } \\
\text { Two-way ANOVA, Turkey's }\end{array}$} & \multirow{2}{*}{$\frac{p \text { value }}{0.0163}$} & \multirow{2}{*}{$\begin{array}{l}F, \text { Dfn, Dfd } \\
\text { Interaction: } 15.96,6,18 ; \text { time point: }\end{array}$} \\
\hline & $1 d$ & $22^{\circ} \mathrm{C}$ vs $15^{\circ} \mathrm{C}$ & & & \\
\hline & & $22^{\circ} \mathrm{C}$ vs $10^{\circ} \mathrm{C}$ & multiple comparison test & 0.0019 & $71.17,3,18$; temperature: $142.8,2,6$ \\
\hline & $2 d$ & $22^{\circ} \mathrm{C}$ vs $15^{\circ} \mathrm{C}$ & & 0.0001 & \\
\hline & & $22^{\circ} \mathrm{C}$ vs $10^{\circ} \mathrm{C}$ & & 0.0001 & \\
\hline & $3 d$ & $22^{\circ} \mathrm{C}$ vs $15^{\circ} \mathrm{C}$ & & 0.0001 & \\
\hline & & $22^{\circ} \mathrm{C}$ vs $10^{\circ} \mathrm{C}$ & & 0.0001 & \\
\hline & $4 \mathrm{~d}$ & $22^{\circ} \mathrm{C}$ vs $15^{\circ} \mathrm{C}$ & & 0.0001 & \\
\hline & & $22^{\circ} \mathrm{C}$ vs $10^{\circ} \mathrm{C}$ & & 0.0001 & \\
\hline \multirow[t]{12}{*}{$1 C$} & $\operatorname{HSP} 90 \alpha$ & $22^{\circ} \mathrm{C}$ vs $15^{\circ} \mathrm{C}$ & Two-way ANOVA, Turkey's & 0.5097 & Interaction: 11.22, 6, 24; Gene: 38.81, \\
\hline & & $22^{\circ} \mathrm{C}$ vs $10^{\circ} \mathrm{C}$ & multiple comparison test & 0.159 & 3,24 ; temperature: $43.31,2,24$ \\
\hline & & $15^{\circ} \mathrm{C}$ vs $10^{\circ} \mathrm{C}$ & & 0.0155 & \\
\hline & HSP90 $\beta$ & $22^{\circ} \mathrm{C}$ vs $15^{\circ} \mathrm{C}$ & & 0.0489 & \\
\hline & & $22^{\circ} \mathrm{C}$ vs $10^{\circ} \mathrm{C}$ & & 0.0024 & \\
\hline & & $15^{\circ} \mathrm{C}$ vs $10^{\circ} \mathrm{C}$ & & 0.4152 & \\
\hline & Grp94 & $22^{\circ} \mathrm{C}$ vs $15^{\circ} \mathrm{C}$ & & $<0.0001$ & \\
\hline & & $22^{\circ} \mathrm{C}$ vs $10^{\circ} \mathrm{C}$ & & $<0.0001$ & \\
\hline & & $15^{\circ} \mathrm{C}$ vs $10^{\circ} \mathrm{C}$ & & 0.0012 & \\
\hline & TRAP-1 & $22^{\circ} \mathrm{C}$ vs $15^{\circ} \mathrm{C}$ & & 0.2602 & \\
\hline & & $22^{\circ} \mathrm{C}$ vs $10^{\circ} \mathrm{C}$ & & 0.1889 & \\
\hline & & $15^{\circ} \mathrm{C}$ vs $10^{\circ} \mathrm{C}$ & & 0.9793 & \\
\hline \multirow[t]{2}{*}{$1 E$} & HSP90 $\beta$ & $22^{\circ} \mathrm{C}$ vs $10^{\circ} \mathrm{C}$ & Unpaired $t$ test with Welch's & 0.7421 & $4.39,2,2$ \\
\hline & Grp94 & $22^{\circ} \mathrm{C}$ vs $10^{\circ} \mathrm{C}$ & correction & 0.0035 & $3.03,2,2$ \\
\hline \multirow[t]{3}{*}{$1 F$} & & Control vs $0.25 \mathrm{~nm}$ & One-way ANOVA, Turkey's & 0.0067 & $75.47,3,12$ \\
\hline & & Control vs $0.5 \mathrm{~nm}$ & multiple comparison test & $<0.0001$ & \\
\hline & & Control vs $1 \mathrm{~nm}$ & & $<0.0001$ & \\
\hline \multirow[t]{2}{*}{$1 H$} & Normalized AChR intensity cluster region & Control vs $17-A A G$ & Unpaired $t$ test & 0.0403 & $4.762,2,2$ \\
\hline & AChR-poor perforations/AChR cluster area & & Unpaired $t$ test & 0.02 & $2.462,3,3$ \\
\hline \multirow[t]{2}{*}{$1 K$} & $24 \mathrm{~h}$ & Control vs 17-AAG & Two-way ANOVA, Sidak's & 0.0274 & Interaction: $0.02911,1,85$; treatment: \\
\hline & $48 \mathrm{~h}$ & & multiple comparison test & 0.0385 & $151.1,1,85$; time point: $6.856,1,85$ \\
\hline \multirow[t]{2}{*}{$1 L$} & $24 \mathrm{~h}$ & Control vs $17-\mathrm{AAG}$ & Two-way ANOVA, Sidak's & 0.9726 & Interaction: $0.5439,1,86$; treatment: \\
\hline & $48 \mathrm{~h}$ & & multiple comparison test & 0.4136 & $80.21,1,86$; time point: $6.856,1,85$ \\
\hline \multirow[t]{2}{*}{$1-1 B$} & Polar metabolites & Control vs $17-\mathrm{AAG}$ & Unpaired $t$ test & Listed in Extended & $\mathrm{N} / \mathrm{A}$ \\
\hline & & & & Data Table 1-1 & \\
\hline \multirow[t]{2}{*}{$1-1 D$} & Fatty acids & & Unpaired $t$ test & Listed in Extended & $\mathrm{N} / \mathrm{A}$ \\
\hline & & & & Data Table 1-2 & \\
\hline \multirow[t]{2}{*}{$1-1 F$} & & Control vs $\mathrm{CHX}$ & One-way ANOVA, Dunnett's & 0.0142 & $9.046,2,6$ \\
\hline & & Control vs $17-A A G$ & multiple comparison test & 0.8498 & \\
\hline $1-1 H$ & & Control vs $17-\mathrm{AAG}$ & $\begin{array}{l}\text { Unpaired } t \text { test with Welch's } \\
\text { correction }\end{array}$ & 0.3747 & $1.388,32,41$ \\
\hline \multirow[t]{22}{*}{$2 B$} & $1 \mathrm{~d}$, Without photobleaching:Control vs $1 \mathrm{~d}$, & aching:17-AAG & Two-way ANOVA, Turkey's & 0.0198 & Interaction: $6.99,3,6$; treatment: \\
\hline & $1 \mathrm{~d}$, Without photobleaching:Control vs $3 \mathrm{~d}$, & aching:Control & multiple comparison test & 0.6093 & $6.916,1,2$; time point: $10.88,3,6$ \\
\hline & $1 \mathrm{~d}$, Without photobleaching:Control vs $3 \mathrm{~d}$, & aching:17-AAG & & 0.0318 & \\
\hline & $1 \mathrm{~d}$, Without photobleaching:Control vs $1 \mathrm{~d}$, & & & 0.0069 & \\
\hline & of aneural AChR cluster:Control & & & & \\
\hline & $1 \mathrm{~d}$, Without photobleachingControl vs $1 \mathrm{~d}$, & & & 0.004 & \\
\hline & of aneural AChR cluster:17-AAG & & & & \\
\hline & $1 \mathrm{~d}$, Without photobleaching:Control vs $3 \mathrm{~d}$, & & & 0.0011 & \\
\hline & of aneural AChR cluster:Control & & & & \\
\hline & $1 \mathrm{~d}$, Without photobleaching:Control vs $3 \mathrm{~d}$, & & & 0.0018 & \\
\hline & of aneural AChR cluster:17-AAG & & & & \\
\hline & $1 \mathrm{~d}$, Without photobleaching:17-AAG vs $3 \mathrm{~d}$ & eaching: & & 0.126 & \\
\hline & Control & & & & \\
\hline & $1 \mathrm{~d}$, Without photobleaching: $17-\mathrm{AAG}$ vs $3 \mathrm{~d}$ & eaching: & & 0.9991 & \\
\hline & 17-AAG & & & & \\
\hline & $1 \mathrm{~d}$, Without photobleaching:17-AAG vs $1 \mathrm{~d}$ & & & 0.8927 & \\
\hline & aneural AChR cluster:Control & & & & \\
\hline & $1 \mathrm{~d}$, Without photobleaching:17-AAG vs $1 \mathrm{~d}$ & & & 0.5569 & \\
\hline & of aneural AChR cluster:17-AAG & & & & \\
\hline & $1 \mathrm{~d}$, Without photobleaching:17-AAG vs $3 \mathrm{~d}$ & & & 0.0818 & \\
\hline & of aneural AChR cluster:Control & & & & \\
\hline & & & & & \\
\hline
\end{tabular}


Table 1: Continued

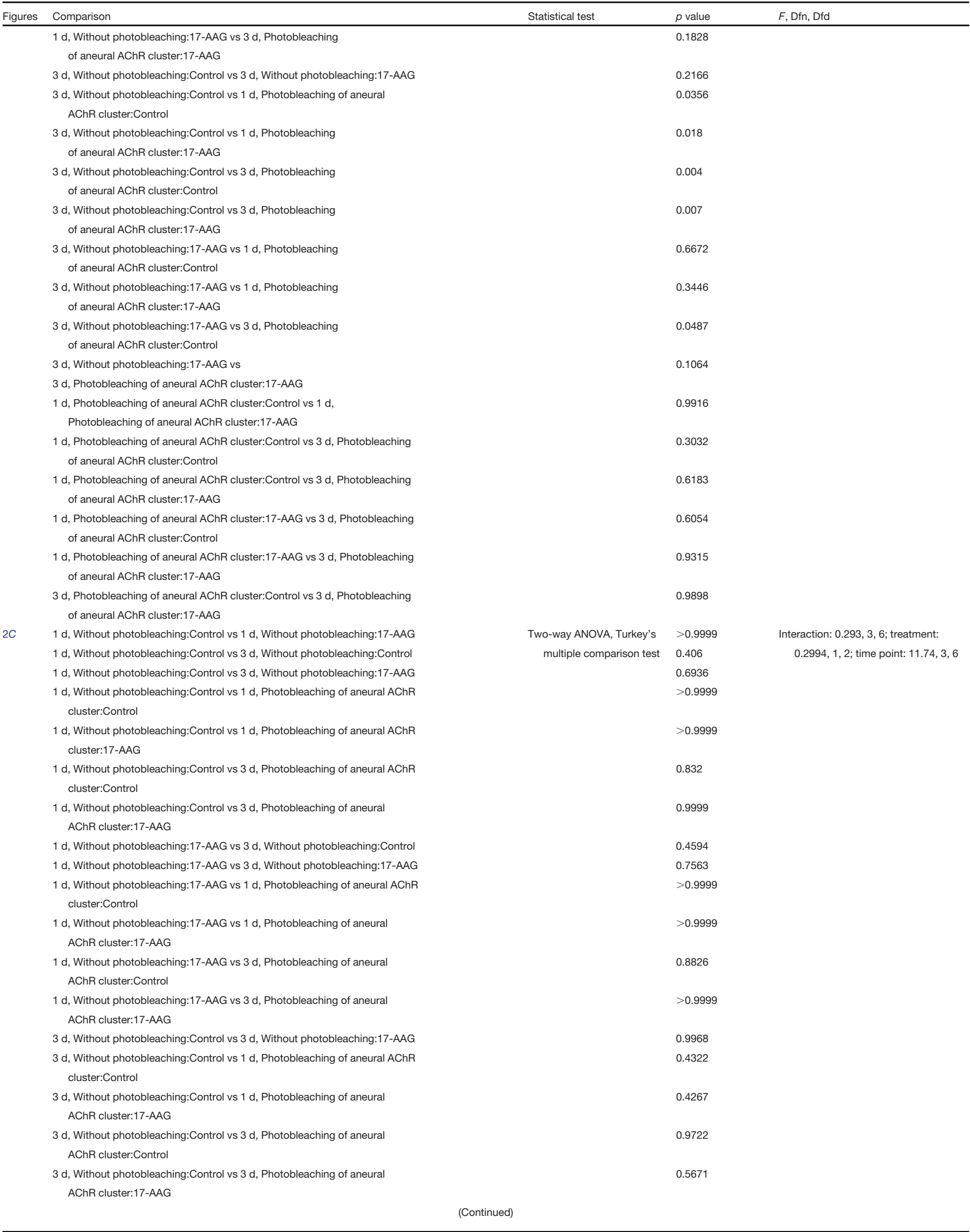


Table 1: Continued

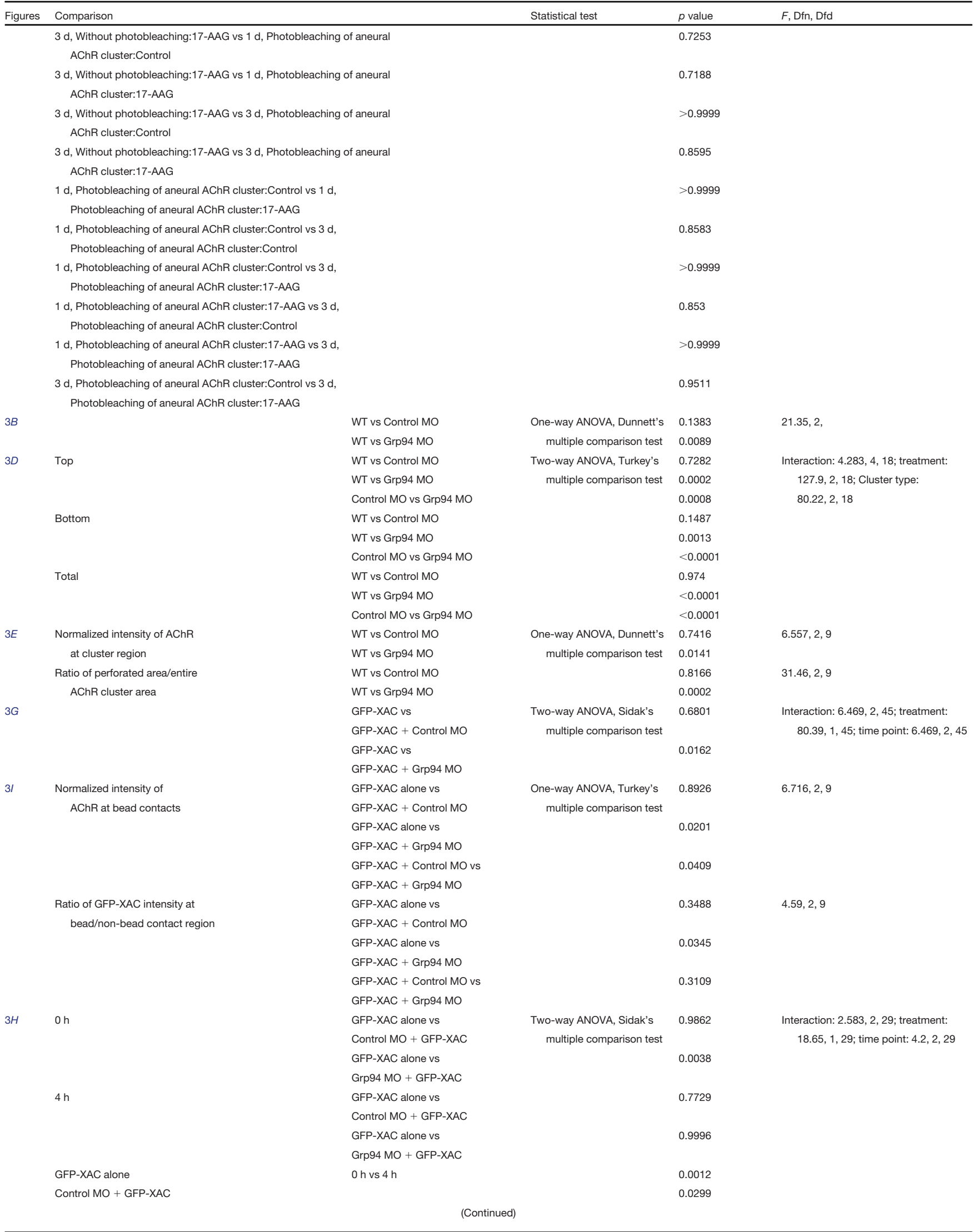


Table 1: Continued

\begin{tabular}{|c|c|c|c|c|c|}
\hline Figures & Comparison & & Statistical test & $p$ value & $F$, Dfn, Dfd \\
\hline & Grp94 MO + GFP-XAC & & & 0.8305 & \\
\hline \multirow{2}{*}{$3-1 B$} & XAC & WT vs Control MO & One-way ANOVA, Dunnett's & 0.1955 & $12.16,2,9$ \\
\hline & & WT vs Grp94 MO & multiple comparison test & 0.0016 & \\
\hline \multirow[t]{2}{*}{$3-1 C$} & p34-Arc & WT vs Control MO & & 0.9667 & $7.445,2,6$ \\
\hline & & WT vs Grp94 MO & & 0.0242 & \\
\hline \multirow[t]{2}{*}{$3-1 D$} & Vinculin & WT vs Control MO & & 0.8551 & $5.924,2,6$ \\
\hline & & WT vs Grp94 MO & & 0.0331 & \\
\hline \multirow[t]{2}{*}{$4 C$} & Perforated region & Control vs $17-\mathrm{AAG}$ & Unpaired $t$ test with Welch's & 0.0025 & $11.1,11,5$ \\
\hline & AChR-rich region & Control vs $17-A A G$ & correction & 0.049 & $11.64,10,5$ \\
\hline \multirow[t]{6}{*}{$4-1 C$} & Perforated region & GFP-XAC vs GFP-XAC (S3A) & One-way ANOVA, Turkey's & 0.1195 & $16.04,2,27$ \\
\hline & & GFP-XAC vs GFP-XAC (S3E) & multiple comparison test & 0.0015 & \\
\hline & & GFP-XAC (S3A) vs GFP-XAC (S3E) & & $<0.0001$ & \\
\hline & AChR-rich region & GFP-XAC vs GFP-XAC (S3A) & & 0.0025 & $16.9,2,27$ \\
\hline & & GFP-XAC vs GFP-XAC (S3E) & & 0.0497 & \\
\hline & & GFP-XAC (S3A) vs GFP-XAC (S3E) & & $<0.0001$ & \\
\hline \multirow[t]{3}{*}{$5 B$} & & WT vs Control MO & One-way ANOVA, Turkey's & 0.511 & $44.59,2,6$ \\
\hline & & WT vs Grp94 MO & multiple comparison test & 0.0003 & \\
\hline & & Control MO vs Grp94 MO & & 0.0007 & \\
\hline \multirow[t]{3}{*}{$5 C$} & & WT vs Control MO & One-way ANOVA, Turkey's & 0.9022 & $8.63,2,6$ \\
\hline & & WT vs Grp94 MO & multiple comparison test & 0.0351 & \\
\hline & & Control MO vs Grp94 MO & & 0.0211 & \\
\hline \multirow[t]{3}{*}{$5 F$} & & WT vs Control MO & Kruskal-Wallis ANOVA test & $\mathrm{N} / \mathrm{A}$ & $\mathrm{N} / \mathrm{A}$ \\
\hline & & WT vs Grp94 MO & with Dunn's multiple & & \\
\hline & & Control MO vs Grp94 MO & comparison test & & \\
\hline \multirow[t]{3}{*}{$5 G$} & & WT vs Control MO & Kruskal-Wallis ANOVA test & & \\
\hline & & WT vs Grp94 MO & with Dunn's multiple & & \\
\hline & & Control MO vs Grp94 MO & comparison test & & \\
\hline \multirow[t]{4}{*}{$5-1 B$} & & Control vs 17-AAG & One-way ANOVA, Dunnett's & 0.0009 & $13.85,4,11$ \\
\hline & & Control vs PU-WS13 & multiple comparison test & 0.0074 & \\
\hline & & Control vs Control MO & & 0.8996 & \\
\hline & & Control vs Grp94 MO & & 0.0004 & \\
\hline \multirow[t]{4}{*}{$5-1 C$} & & Control vs $17-\mathrm{AAG}$ & & 0.0195 & $5.745,4,11$ \\
\hline & & Control vs PU-WS13 & & 0.0233 & \\
\hline & & Control vs Control MO & & 0.9971 & \\
\hline & & Control vs Grp94 MO & & 0.0329 & \\
\hline \multirow[t]{4}{*}{$5-2 B$} & $0.5 \mathrm{~h}$ & Control vs $17-\mathrm{AAG}$ & One-way ANOVA, Dunnett's & 0.0001 & $9.548,2,74$ \\
\hline & & Control vs PU-WS13 & multiple comparison test & 0.0154 & \\
\hline & $4 \mathrm{~h}$ & Control vs $17-\mathrm{AAG}$ & & 0.0001 & $20.28,2,73$ \\
\hline & & Control vs PU-WS13 & & 0.0001 & \\
\hline
\end{tabular}

\section{References}

Bader DM, Wilkening S, Lin G, Tekkedil MM, Dietrich K, Steinmetz LM, Gagneur J (2015) Negative feedback buffers effects of regulatory variants. Mol Syst Biol 11:785.

Bernstein BW, Bamburg JR (2010) ADF/cofilin: a functional node in cell biology. Trends Cell Biol 20:187-195.

Borges LS, Ferns M (2001) Agrin-induced phosphorylation of the acetylcholine receptor regulates cytoskeletal anchoring and clustering. J Cell Biol 153:1-12.

Brennan GP, Jimenez-Mateos EM, McKiernan RC, Engel T, Tzivion G, Henshall DC (2013) Transgenic overexpression of 14-3-3 zeta protects hippocampus against endoplasmic reticulum stress and status epilepticus in vivo. PLoS One 8:e54491.

Bruneau EG, Brenner DS, Kuwada JY, Akaaboune M (2008) Acetylcholine receptor clustering is required for the accumulation and maintenance of scaffolding proteins. Curr Biol 18:109-115.

Chan ZC, Oentaryo MJ, Lee CW (2020a) MMP-mediated modulation of ECM environment during axonal growth and NMJ development. Neurosci Lett 724:134822.

Chan ZC, Kwan HR, Wong YS, Jiang Z, Zhou Z, Tam KW, Chan YS, Chan CB, Lee CW (2020b) Site-directed MT1-MMP trafficking and surface insertion regulate $\mathrm{AChR}$ clustering and remodeling at developing NMJs. Elife 9:e54379.
Chong J, Wishart DS, Xia J (2019) Using MetaboAnalyst 4.0 for comprehensive and integrative metabolomics data analysis. Curr Protoc Bioinformatics 68:e86.

Chugh P, Paluch EK (2018) The actin cortex at a glance. J Cell Sci 131:jcs186254.

Csermely P, Schnaider T, Soti C, Prohászka Z, Nardai G (1998) The 90-kDa molecular chaperone family: structure, function, and clinical applications. A comprehensive review. Pharmacol Ther 79:129-168.

Dai Z, Peng HB (1998) A role of tyrosine phosphatase in acetylcholine receptor cluster dispersal and formation. J Cell Biol 141:16131624.

Dai Z, Luo X, Xie H, Peng HB (2000) The actin-driven movement and formation of acetylcholine receptor clusters. J Cell Biol 150:13211334.

DiDomenico BJ, Bugaisky GE, Lindquist S (1982) The heat shock response is self-regulated at both the transcriptional and posttranscriptional levels. Cell 31:593-603.

Dobbins GC, Luo S, Yang Z, Xiong WC, Mei L (2008) Alpha-actinin interacts with rapsyn in agrin-stimulated AChR clustering. Mol Brain 1:18.

Fotedar R, Margolis RL (2015) WISp39 and Hsp90: actin' together in cell migration. Oncotarget 6:17871-17872. 
Frasson M, Vitadello M, Brunati AM, La Rocca N, Tibaldi E, Pinna LA, Gorza L, Donella-Deana A (2009) Grp94 is Tyr-phosphorylated by Fyn in the lumen of the endoplasmic reticulum and translocates to Golgi in differentiating myoblasts. Biochim Biophys Acta 1793:239-252.

Geng L, Zhang HL, Peng HB (2009) The formation of acetylcholine receptor clusters visualized with quantum dots. BMC Neurosci 10:80.

Gutiérrez T, Simmen T (2014) Endoplasmic reticulum chaperones and oxidoreductases: critical regulators of tumor cell survival and immunorecognition. Front Oncol 4:291.

Haghighi AP, McCabe BD, Fetter RD, Palmer JE, Hom S, Goodman CS (2003) Retrograde control of synaptic transmission by postsynaptic CaMKII at the Drosophila neuromuscular junction. Neuron 39:255-267.

Hobson BM (1965) Cold blooded vertebrates, including Xenopus laevis. Food Cosmet Toxicol 3:209-215.

Iwasa K, Nambu Y, Motozaki Y, Furukawa Y, Yoshikawa H, Yamada M (2014) Increased skeletal muscle expression of the endoplasmic reticulum chaperone GRP78 in patients with myasthenia gravis. J Neuroimmunol 273:72-76.

Kummer TT, Misgeld T, Lichtman JW, Sanes JR (2004) Nerve-independent formation of a topologically complex postsynaptic apparatus. J Cell Biol 164:1077-1087.

Lee CW, Han J, Bamburg JR, Han L, Lynn R, Zheng JQ (2009) Regulation of acetylcholine receptor clustering by ADF/cofilin-directed vesicular trafficking. Nat Neurosci 12:848-856.

Lee CW, Zhang H, Geng L, Peng HB (2014) Crosslinking-induced endocytosis of acetylcholine receptors by quantum dots. PLoS One 9:e90187.

Li L, Cao Y, Wu H, Ye X, Zhu Z, Xing G, Shen C, Barik A, Zhang B, Xie X, Zhi W, Gan L, Su H, Xiong WC, Mei L (2016) Enzymatic activity of the scaffold protein rapsyn for synapse formation. Neuron 92:1007-1019.

Li L, Xiong WC, Mei L (2018) Neuromuscular junction formation, aging, and disorders. Annu Rev Physiol 80:159-188.

Li XM, Dong XP, Luo SW, Zhang B, Lee DH, Ting AK, Neiswender $\mathrm{H}$, Kim CH, Carpenter-Hyland E, Gao TM, Xiong WC, Mei L (2008) Retrograde regulation of motoneuron differentiation by muscle beta-catenin. Nat Neurosci 11:262-268.

Lin W, Burgess RW, Dominguez B, Pfaff SL, Sanes JR, Lee KF (2001) Distinct roles of nerve and muscle in postsynaptic differentiation of the neuromuscular synapse. Nature 410:1057-1064.

Liu B, Staron M, Hong F, Wu BX, Sun S, Morales C, Crosson CE, Tomlinson S, Kim I, Wu D, Li Z (2013) Essential roles of grp94 in gut homeostasis via chaperoning canonical Wnt pathway. Proc Natl Acad Sci USA 110:6877-6882.

Liu J, Xu Y, Stoleru D, Salic A (2012) Imaging protein synthesis in cells and tissues with an alkyne analog of puromycin. Proc Natl Acad Sci USA 109:413-418.

Luo S, Zhang B, Dong XP, Tao Y, Ting A, Zhou Z, Meixiong J, Luo J, Chiu FC, Xiong WC, Mei L (2008) HSP90 beta regulates rapsyn turnover and subsequent AChR cluster formation and maintenance. Neuron 60:97-110.

Marzec M, Eletto D, Argon Y (2012) GRP94: an HSP90-like protein specialized for protein folding and quality control in the endoplasmic reticulum. Biochim Biophys Acta 1823:774-787.

Murphy N, Bonner HP, Ward MW, Murphy BM, Prehn JH, Henshall DC (2008) Depletion of 14-3-3 zeta elicits endoplasmic reticulum stress and cell death, and increases vulnerability to kainate-induced injury in mouse hippocampal cultures. J Neurochem 106:978-988.

Oury J, Liu Y, Töpf A, Todorovic S, Hoedt E, Preethish-Kumar V, Neubert TA, Lin W, Lochmüller H, Burden SJ (2019) MACF1 links Rapsyn to microtubule- and actin-binding proteins to maintain neuromuscular synapses. J Cell Biol 218:1686-1705.
Peng HB, Baker LP, Chen Q (1991) Tissue culture of Xenopus neurons and muscle cells as a model for studying synaptic induction. Methods Cell Biol 36:511-526.

Prodromou C (2016) Mechanisms of Hsp90 regulation. Biochem J 473:2439-2452.

Proszynski TJ, Gingras J, Valdez G, Krzewski K, Sanes JR (2009) Podosomes are present in a postsynaptic apparatus and participate in its maturation. Proc Natl Acad Sci USA 106:18373-18378.

Richter K, Haslbeck M, Buchner J (2010) The heat shock response: life on the verge of death. Mol Cell 40:253-266.

Rizzo AM, Adorni L, Montorfano G, Rossi F, Berra B (2007) Antioxidant metabolism of Xenopus laevis embryos during the first days of development. Comp Biochem Physiol B Biochem Mol Biol 146:94-100.

Sanes JR, Lichtman JW (2001) Induction, assembly, maturation and maintenance of a postsynaptic apparatus. Nat Rev Neurosci 2:791-805.

Schopf FH, Biebl MM, Buchner J (2017) The HSP90 chaperone machinery. Nat Rev Mol Cell Biol 18:345-360.

Sharp S, Workman P (2006) Inhibitors of the HSP90 molecular chaperone: current status. Adv Cancer Res 95:323-348.

Slomnicki LP, Pietrzak M, Vashishta A, Jones J, Lynch N, Elliot S, Poulos E, Malicote D, Morris BE, Hallgren J, Hetman M (2016) Requirement of neuronal ribosome synthesis for growth and maintenance of the dendritic tree. J Biol Chem 291:5721-5739.

Suzuki S, Utsugisawa K, Iwasa K, Satoh T, Nagane Y, Yoshikawa H, Kuwana M, Suzuki N (2011) Autoimmunity to endoplasmic reticulum chaperone GRP94 in myasthenia gravis. J Neuroimmunol 237:87-92.

Tsai PI, Wang M, Kao HH, Cheng YJ, Lin YJ, Chen RH, Chien CT (2012) Activity-dependent retrograde laminin A signaling regulates synapse growth at Drosophila neuromuscular junctions. Proc Natl Acad Sci USA 109:17699-17704.

Van PN, Peter F, Söling HD (1989) Four intracisternal calcium-binding glycoproteins from rat liver microsomes with high affinity for calcium. No indication for calsequestrin-like proteins in inositol 1,4,5-trisphosphate-sensitive calcium sequestering rat liver vesicles. J Biol Chem 264:17494-17501.

Vogl AM, Phu L, Becerra R, Giusti SA, Verschueren E, Hinkle TB, Bordenave MD, Adrian M, Heidersbach A, Yankilevich P, Stefani FD, Wurst W, Hoogenraad CC, Kirkpatrick DS, Refojo D, Sheng M (2020) Global site-specific neddylation profiling reveals that NEDDylated cofilin regulates actin dynamics. Nat Struct Mol Biol 27:210-220.

Wang ZZ, Mathias A, Gautam M, Hall ZW (1999) Metabolic stabilization of muscle nicotinic acetylcholine receptor by rapsyn. J Neurosci 19:1998-2007.

Xing G, Xiong WC, Mei L (2020) Rapsyn as a signaling and scaffolding molecule in neuromuscular junction formation and maintenance. Neurosci Lett 731:135013.

Yang X, Li W, Prescott ED, Burden SJ, Wang JC (2000) DNA topoisomerase Ilbeta and neural development. Science 287:131134.

Yang X, Arber S, William C, Li L, Tanabe Y, Jessell TM, Birchmeier C, Burden SJ (2001) Patterning of muscle acetylcholine receptor gene expression in the absence of motor innervation. Neuron 30:399-410.

Yeates CJ, Zwiefelhofer DJ, Frank CA (2017) The maintenance of synaptic homeostasis at the Drosophila neuromuscular junction is reversible and sensitive to high temperature. eNeuro 4:ENEURO.022017.2017.

Yeo HL, Lim JY, Fukami Y, Yuki N, Lee CW (2015) Using Xenopus tissue cultures for the study of myasthenia gravis pathogenesis. Dev Biol 408:244-251.

Zhu Y, de Castro L, Cooper RL (2018) Effect of temperature change on synaptic transmission at crayfish neuromuscular junctions. Biol Open 7:bio037820. 